\title{
An iterative method for computing the pseudospectral abscissa for a class of nonlinear eigenvalue problems
}

\author{
Wim Michiels Nicola Guglielmi
}

Report TW607, January 2012

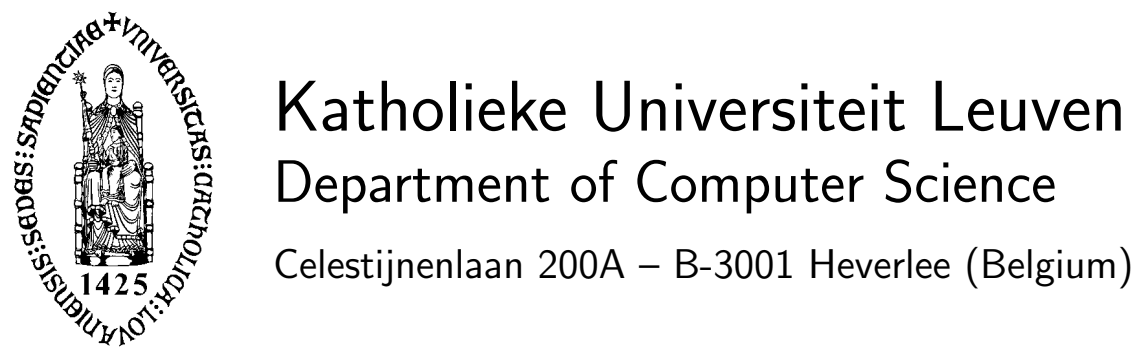




\title{
An iterative method for computing the pseudospectral abscissa for a class of nonlinear eigenvalue problems
}

\author{
Wim Michiels Nicola Guglielmi \\ Report TW60\%, January 2012 \\ Department of Computer Science, K.U.Leuven
}

Abstract

We consider the following class of nonlinear eigenvalue problems,

$$
\left(\sum_{i=1}^{m} A_{i} p_{i}(\lambda)\right) v=0,
$$

where $A_{1}, \ldots, A_{m}$ are given $n \times n$ matrices and the functions $p_{1}, \ldots, p_{m}$ are assumed to be entire. This does not only include polynomial eigenvalue problems but also eigenvalue problems arising from systems of delay differential equations. Our aim is to compute the $\epsilon$-pseudospectral abscissa, i.e. the real part of the rightmost point in the $\epsilon$-pseudospectrum, which is the complex set obtained by joining all solutions of the eigenvalue problem under perturbations $\left\{\delta A_{i}\right\}_{i=1}^{m}$, of norm at most $\epsilon$, of the matrices $\left\{A_{i}\right\}_{i=1}^{m}$.

In analogy to the linear eigenvalue problem we prove that it is sufficient to restrict the analysis to rank-1 perturbations of the form $\delta A_{i}=\beta_{i} u v^{*}$ where $u \in \mathbb{C}^{n}$ and $v \in \mathbb{C}^{n}$ with $\beta_{i} \in$ $\mathbb{C}$ for all $i$. Using this main - and unexpected - result we present new iterative algorithms which only require the computation of the spectral abscissa of a sequence of problems obtained by adding rank one updates to the matrices $A_{i}$. These provide lower bounds to the pseudspectral abscissa and in most cases converge to it. A detailed analysis of the convergence of the algorithms is made.

The methods available for the standard eigenvalue problem in the literature provide a robust and reliable computation but at the cost of full eigenvalue decompositions of order $2 n$ and singular value decompositions, making them unfeasible for large systems. Moreover, these methods cannot be generalized to nonlinear eigenvalue problems, as we shall explain. Therefore, the presented method is the first generally applicable method for nonlinear problems. In order to be applied it simply requires a procedure to compute the rightmost eigenvalue and the corresponding left and right eigenvectors. In addition, if the matrices $A_{i}$ are large and sparse then the computation of the rightmost eigenvalue can for many classes of nonlinear eigenvalue problems be performed in an efficient way by iterative algorithms which only rely on matrix vector multiplication and on solving systems of linear equations, where the structure of the matrices (original sparse matrices plus rank one updates) can be exploited. This feature, as well other properties of the presented numerical methods, are illustrated by means of the delay and polynomial eigenvalue problem.

Keywords : Pseudospectrum, nonlinear eigenvalue problem, pseudospectral abscissa, stability of dynamical systems, sparse matrices 


\title{
AN ITERATIVE METHOD FOR COMPUTING THE PSEUDOSPECTRAL ABSCISSA FOR A CLASS OF NONLINEAR EIGENVALUE PROBLEMS
}

\author{
WIM MICHIELS* AND NICOLA GUGLIELMI ${ }^{\dagger}$
}

Abstract. We consider the following class of nonlinear eigenvalue problems,

$$
\left(\sum_{i=1}^{m} A_{i} p_{i}(\lambda)\right) v=0,
$$

where $A_{1}, \ldots, A_{m}$ are given $n \times n$ matrices and the functions $p_{1}, \ldots, p_{m}$ are assumed to be entire. This does not only include polynomial eigenvalue problems but also eigenvalue problems arising from systems of delay differential equations. Our aim is to compute the $\epsilon$-pseudospectral abscissa, i.e. the real part of the rightmost point in the $\epsilon$-pseudospectrum, which is the complex set obtained by joining all solutions of the eigenvalue problem under perturbations $\left\{\delta A_{i}\right\}_{i=1}^{m}$, of norm at most $\epsilon$, of the matrices $\left\{A_{i}\right\}_{i=1}^{m}$.

In analogy to the linear eigenvalue problem we prove that it is sufficient to restrict the analysis to rank-1 perturbations of the form $\delta A_{i}=\beta_{i} u v^{*}$ where $u \in \mathbb{C}^{n}$ and $v \in \mathbb{C}^{n}$ with $\beta_{i} \in \mathbb{C}$ for all $i$. Using this main - and unexpected - result we present new iterative algorithms which only require the computation of the spectral abscissa of a sequence of problems obtained by adding rank one updates to the matrices $A_{i}$. These provide lower bounds to the pseudspectral abscissa and in most cases converge to it. A detailed analysis of the convergence of the algorithms is made.

The methods available for the standard eigenvalue problem in the literature provide a robust and reliable computation but at the cost of full eigenvalue decompositions of order $2 n$ and singular value decompositions, making them unfeasible for large systems. Moreover, these methods cannot be generalized to nonlinear eigenvalue problems, as we shall explain. Therefore, the presented method is the first generally applicable method for nonlinear problems. In order to be applied it simply requires a procedure to compute the rightmost eigenvalue and the corresponding left and right eigenvectors. In addition, if the matrices $A_{i}$ are large and sparse then the computation of the rightmost eigenvalue can for many classes of nonlinear eigenvalue problems be performed in an efficient way by iterative algorithms which only rely on matrix vector multiplication and on solving systems of linear equations, where the structure of the matrices (original sparse matrices plus rank one updates) can be exploited. This feature, as well other properties of the presented numerical methods, are illustrated by means of the delay and polynomial eigenvalue problem.

Keywords. Pseudospectrum, nonlinear eigenvalue problem, pseudospectral abscissa, stability of dynamical systems, sparse matrices.

1. Introduction. Characterizing and computing pseudospectra are well established tools for analyzing the sensitivity of eigenvalues of a system [23]. Pseudospectra are sets in the complex plane to which the eigenvalues can be shifted when the system is subject to perturbations with a specified upper bound. For linear autonomous dynamical systems the position of the spectrum associated with the differential equation or evolutionary operator gives valuable information about the dynamic behavior, whenever the solutions satisfy a spectrum determined growth property. In particular, in the continuous-time case the spectral abscissa determines the asymptotic growth rate of the solutions as well as the stability properties of the equilibrium. Similarly, if a system is subject to uncertainty, the pseudospectral abscissa constitutes a bound on the asymptotic growth rate of the solutions of the perturbed system, which is uniform over all possible perturbations under consideration, and it allows to assess the stability robustness [11]. The pseudospectral abscissa (or its counter part in the

*Department of Computer Science, Katholieke Universiteit Leuven, Celestijnenlaan 200A, 3001 Heverlee, Belgium (Wim.Michiels@cs.kuleuven.be).

†Dipartimento di Matematica Pura e Applicata, Università dell'Aquila, via Vetoio (Coppito), I-67010 L'Aquila, Italy (guglielm@univaq.it). 
discrete-time case, the pseudospectral radius) is hence closely related to the distance to instability [7] and to the $\mathcal{H}_{\infty}$ norm of appropriately defined transfer functions (see, e.g., [26] for relations between $\mathcal{H}_{\infty}$ norms and robust stability criteria). The position of pseudospectra can also be used to predict the transient behavior of solutions of differential or difference equations, and, in this way, it allows for instance to explain the behavior of certain iterative methods [23].

In this article we consider the pseudospectral abscissa computation for a broad class of nonlinear eigenvalue problems. This class includes polynomial eigenvalue problems and nonlinear eigenvalue problems arising in the analysis of linear delay differential equations as special cases. Although many of these problems can be reformulated as linear eigenvalue problems (for example, by a so-called linearization of a polynomial eigenvalue problem or by a transformation to an equivalent operator eigenvalue problem for the delay eigenvalue problem [18]) we will not consider the unstructured pseudospectra of a particular type of linearization (note that the pseudospectra strongly depend on the basis). Instead, as in [17, 21], we will explicitly take the structure of the original nonlinear eigenvalue problem into account in the definition of pseudospectra, which we relate to the effect of perturbations on the individual coefficient matrices. Mathematically, the general setting is the nonlinear eigenvalue problem

$$
\left(\sum_{i=1}^{m} A_{i} p_{i}(\lambda)\right) v=0, \lambda \in \mathbb{C}, v \in \mathbb{C}^{n},
$$

where $A_{i} \in \mathbb{C}^{n \times n}, i=1, \ldots, m$ and the functions $p_{i}: \mathbb{C} \rightarrow \mathbb{C}, i=1, \ldots, m$ are entire and satisfy $p_{i}(\bar{\lambda})=\overline{p_{i}(\lambda)}, 1 \leq i \leq m$. In what follows we call $\left(\sum_{i=1}^{m} A_{i} p_{i}(\lambda)\right)$ the characteristic matrix. We denote the spectrum by $\Lambda$, i.e.

$$
\Lambda:=\left\{\lambda \in \mathbb{C}: \operatorname{det}\left(\sum_{i=1}^{m} A_{i} p_{i}(\lambda)\right)=0\right\} .
$$

We are interested in the effect of bounded perturbations of the matrices $A_{i}$ on the spectrum, which leads to the perturbed eigenvalue problem,

$$
\left(\sum_{i=1}^{m}\left(A_{i}+\delta A_{i}\right) p_{i}(\lambda)\right) v=0, \lambda \in \mathbb{C}, v \in \mathbb{C}^{n} .
$$

The first step in the robustness analysis is to define the class of perturbations under consideration, as well as a measure of the combined perturbation

$$
\Delta:=\left(\delta A_{1}, \ldots, \delta A_{m}\right) .
$$

In this work, in analogy to the classical definition of $\epsilon$-pseudospectrum of a matrix [23], we allow the perturbations $\delta A_{i}, i=1, \ldots, m$, to be complex matrices, i.e.,

$$
\Delta \in \mathbb{C}^{n \times n \times m} \text {. }
$$

Introducing weights $w_{i} \in \overline{\mathbb{R}}_{0}^{+}, i=1, \ldots, m$, where $\overline{\mathbb{R}}_{0}^{+}=\mathbb{R}^{+} \backslash\{0\} \cup\{\infty\}$, we define the following global measure of the perturbations:

$$
\|\Delta\|_{\text {glob }}:=\left\|\left[\begin{array}{c}
w_{1}\left\|\delta A_{1}\right\|_{2} \\
\vdots \\
w_{m}\left\|\delta A_{m}\right\|_{2}
\end{array}\right]\right\|_{\infty} .
$$


In this way the condition

$$
\|\Delta\|_{\text {glob }} \leq \epsilon
$$

corresponds to the natural assumptions of taking perturbations satisfying

$$
\left\|\delta A_{i}\right\|_{2} \leq \epsilon / w_{i}, i=1, \ldots, m
$$

This uncertainty bound is also used in [21] and fits within the general class considered in [17]. Taking a weight equal to infinity implies that the corresponding matrix is not perturbed.

With the above class of allowable perturbations and with the measure (1.3) we define the $\epsilon$-pseudospectrum of (1.1) as the set

$$
\Lambda_{\epsilon}=\bigcup_{\|\Delta\|_{\text {glob } \leq \epsilon}}\left\{\lambda \in \mathbb{C}: \operatorname{det}\left(\sum_{i=1}^{m}\left(A_{i}+\delta A_{i}\right) p_{i}(\lambda)\right)=0\right\}
$$

where $\Delta=\left(\delta A_{1}, \ldots, \delta A_{m}\right) \in \mathbb{C}^{n \times n \times m}$, and we define the corresponding pseudospectral abscissa as

$$
\alpha_{\epsilon}:=\sup \left\{\Re(\lambda): \lambda \in \Lambda_{\epsilon}\right\} \text {. }
$$

Note that if a stability notion of a dynamical system is associated with the requirement that the spectrum be located in the open left half plane and bounded away from the imaginary axis, than the distance to instability of a stable system can be expressed as

$$
d:=\inf \left\{\epsilon>0: \alpha_{\epsilon} \geq 0\right\}
$$

In [17, Theorem 1] the following explicit expression for the $\epsilon$-pseudospectrum was stated.

Proposition 1.1. For the perturbation measure (1.3) the pseudospectrum $\Lambda_{\epsilon}$ satisfies

$$
\Lambda_{\epsilon}=\left\{\lambda \in \mathbb{C}: \sigma_{n}\left(\sum_{i=1}^{m} A_{i} p_{i}(\lambda)\right) \leq \epsilon\|w(\lambda)\|_{1},\right\}
$$

where

$$
w(\lambda)=\left[\begin{array}{lll}
\frac{p_{0}(\lambda)}{w_{0}} & \cdots & \frac{p_{m}(\lambda)}{w_{m}}
\end{array}\right]^{T} .
$$

Observe that in (1.6) all information about the structure of the uncertainty on the characteristic matrix is contained in the factor

$$
\|w(\lambda)\|_{1}=\sum_{i=1}^{m} \frac{\left|p_{i}(\lambda)\right|}{w_{i}}
$$

For the linear eigenvalue problem level set methods are well established methods for computing the distance to instability and related quantities such as $\mathcal{H}_{\infty}$ norms and pseudospectral abscissa. For the latter, the underlying idea is that the intersections in the complex plane between an $\epsilon$-pseudospectrum and the line $\Re(\lambda)=c$ or $\Im(\lambda)=c$, 
with $c$ an arbitrary real constant, can be directly computed from the solutions of a structured eigenvalue problem. This idea lays at the basis of the quadratically converging algorithm for computing the pseudospectral abscissa in [6], where the rightmost point on the pseudospectrum is located using a two-directional, crisscross search. This algorithm resembles the algorithms for computing $\mathcal{H}_{\infty}$ norms presented in $[2,4]$. Although level set methods have proven to be very robust, they require in each iteration step the computation of all eigenvalues on the real or imaginary axis of a matrix of twice the size of the original problem and additional singular value decompositions, which makes them prohibitively expensive for large problems. A different approach has recently been proposed in [11], which relies on the property that eigenvalues can be shifted to the boundary of the pseudospectrum by rank-one perturbations of the matrix and on the characterization of extrema of the pseudospectra as fixed points of an iteration map. The main distinction with level set methods is that the algorithm of [11] only requires the computation of the rightmost eigenvalue of matrices obtained by adding rank-one perturbations to the original matrix, for which -in case of large and sparse problems- efficient iterative solvers can be used. The computational cost usually amounts to a constant times the cost of computing the spectral abscissa [11].

For nonlinear eigenvalue problems there are merely methods available in the literature (besides the brute-force approach of computing pseudospectra using a grid on the complex plane). Two aspects are important in generalizing results for linear eigenvalue problems. First, in most cases a direct generalization of level-set methods is not possible. This can be explained by the presence of the extra factor (1.8) in (1.6), which prevents the intersection of the pseudospectrum and horizontal /vertical lines to be characterized by the solutions of an eigenvalue problem. An exception is the delay eigenvalue problem as considered in [12], where the intersection of pseudospectra with the line $\Re(\lambda)=c$ can be found by solving a Hamiltonian eigenvalue problem, following from the property that (1.8) is constant on the line $\Re(\lambda)=c$ if $p_{i}=\exp \left(-\lambda \tau_{i}\right)$. However, since in the "horizontal direction" a similar argument is not applicable, a (linearly converging) bisection search is needed to locate the rightmost point on the pseudospectrum. The large number of iteration steps, as well as the fact that the Hamiltonian eigenvalue problem is infinite-dimensional and needs to be discretized, makes the overall algorithm of [12] computationally demanding.

Second, a linearization of the nonlinear eigenvalue problem or an approximation that results in a standard eigenvalue problem, followed by the pseudospectral abscissa computation using the previously described methods, does in general not solve the problem because the structure of the perturbations, inherited from the original nonlinear equation, is not respected.

The algorithms presented in this paper build on a combination of results in [17] and [11] (see also [10]). As a starting point we show in Proposition 3.1 that the boundary of the pseudospectra, as defined by (1.4), remains invariant if the allowable perturbations on the matrices $A_{i}, 1 \leq i \leq m$, are further restricted to be rank-one matrices of the form $\delta A_{i}=\beta_{i} u v^{*}$ where $\beta_{i} \in \mathbb{C}$ for all $i$ and $u, v \in \mathbb{C}^{n}$ not depending on $i$. This means that all perturbations can be considered to be proportional to a common rank-one matrix. This key and unexpected property allows us to generalize the ideas of [11]. Similarly to the case of the standard eigenvalue problem, the application of the proposed algorithm only requires the availability of a method for the computation of the rightmost eigenvalue. For several classes of nonlinear eigenvalue problems, methods and software are available. See for instance [22] and the references therein for an overview on the quadratic eigenvalue problem and $[3,8,14]$ for the delay eigenvalue 
problem. General purpose methods for solving nonlinear eigenvalue problems of the form (1.1) are described in, e.g., [15, 24, 16, 25] and the references therein.

The structure of the paper is as follows. In Section 2 the generality of the setting is illustrated and technical assumptions are made. Section 3 describes the algorithm for the pseudospectral abscissa computation. In Sections 4 and 5 a convergence analysis is presented, and improvements are discussed. Numerical experiments are described in Section 6. The conclusions are presented in Section 7. In Appendix A the generalization of the results of the paper to other perturbation measures than (1.3) is outlined.

\section{Notation.}

$\mathbb{C}, \mathbb{R}$

$\mathbb{N}$

$\mathbb{R}^{+}, \mathbb{R}_{0}^{+}$

$A^{T}, A^{*}$

$\rho(A)$

$\sigma_{1}(A) \geq \sigma_{2}(A) \geq \cdots$

$I, I_{n}$

$\alpha(M), \quad M: \mathbb{C} \rightarrow \mathbb{C}^{n \times n}$

$\Re(u), \Im(u), \bar{u}$, with $u \in \mathbb{C}$ $\partial S$, with $S \subset C$
: the imaginary unit

: set of the complex and real numbers

: set of natural numbers

: set of nonnegative and strictly positive real numbers

: transpose, complex conjugate transpose of matrix $A$

: spectral radius of matrix $A$

: singular values of matrix $A$

: identity matrix of appropriate dimensions,

of dimensions $n \times n$

: spectral abscissa, $\alpha(M)=\sup _{\lambda \in \mathbb{C}}\{\Re(\lambda): \operatorname{det} M(\lambda)=0\}$

: real part, imaginary part, complex conjugate of $u$ : boundary of the set $S$

2. Examples and assumptions. Let us first illustrate how several classes of perturbed eigenvalue problem fit within the framework of the paper. The perturbed standard eigenvalue problem

$$
(\lambda I-(A+\delta A)) v=0
$$

is of the form (1.2) with $m=2, p_{1}(\lambda)=\lambda, p_{2}(\lambda)=-1, A_{0}=I, A_{1}=-A$. To express that the first term is not perturbed we can set $w_{1}=\infty$. The polynomial eigenvalue problem

$$
\left(\sum_{i=1}^{m}\left(A_{i}+\delta A_{i}\right) \lambda^{i-1}\right) v=0
$$

is also of the form (1.2). With all weights equal to 1, expression (1.6) becomes

$$
\Lambda_{\epsilon}=\left\{\lambda \in \mathbb{C}: \sigma_{n}\left(\sum_{i=1}^{m} A_{i} \lambda^{i-1}\right) /\left(\sum_{i=1}^{m}|\lambda|^{i-1}\right) \leq \epsilon\right\},
$$

which is in accordance with [21]. As a final example the delay eigenvalue problem

$$
\left(\lambda I-\left(B_{0}+\delta B_{0}\right)-\sum_{i=1}^{\ell}\left(B_{i}+\delta B_{i}\right) e^{-\lambda \tau_{i}}\right) v=0
$$


can be analyzed by taking $m=\ell+2, p_{0}(\lambda)=\lambda, p_{1}(\lambda)=-1, p_{i}(\lambda)=-\exp \left(-\lambda \tau_{i}\right), 1 \leq$ $i \leq \ell$, and setting $w_{1}=\infty$. Taking weights $w_{2}=\cdots=w_{m}=1$, expression (1.6) becomes

$$
\Lambda_{\epsilon}=\left\{\lambda \in \mathbb{C}: \sigma_{n}\left(\lambda I-B_{0}-\sum_{i=1}^{\ell} B_{i} e^{-\lambda \tau_{i}}\right) /\left(1+\sum_{i=1}^{\ell}\left|e^{-\lambda \tau_{i}}\right|\right) \leq \epsilon\right\} .
$$

For nonlinear eigenvalue problems in their most general form (1.1) the pseudospectral abscissa may be equal to infinity, or a globally rightmost point of the pseudospectrum may not exist. Since the algorithm presented in the next section relies on the explicit computation of a globally rightmost point, we make the following assumption throughout the paper, which guarantees its existence.

Assumption 2.1. For all $r \in \mathbb{R}$ the set $\Lambda_{\epsilon} \cap\{\lambda \in \mathbb{C}: \Re(\lambda) \geq r\}$ is bounded.

For linear eigenvalue problems and for eigenvalue problems inferred from linear time-delay systems of retarded type, which includes (2.2), Assumption 2.1 is satisfied for all values of $\epsilon>0$ (see $[9,18]$ ). For polynomial eigenvalue problems the situation is different, as illustrated with the following result.

Proposition 2.2. Consider eigenvalue problem (2.1). Assume that matrix $A_{m}$ is invertible. If $\epsilon<w_{m} \sigma_{n}\left(A_{m}\right)$ then $\Lambda_{\epsilon}$ is bounded. If $\epsilon>w_{m} \sigma_{n}\left(A_{m}\right)$ then $\Lambda_{\epsilon}$ is unbounded and, in addition, $\alpha_{\epsilon}=+\infty$.

The bound on $\epsilon$ stems from the fact that eigenvalues are shifted to infinity if a perturbation renders the leading coefficient matrix singular.

3. Computing the pseudospectral abscissa. Before presenting the algorithm in $§ 3.2$ we first give the main theoretical results.

3.1. Theoretical foundation. The following proposition characterize points lying on the boundary of pseudospectra contours.

Proposition 3.1. Let $\lambda_{\epsilon} \in \mathbb{C}$ and consider the statements:

(i) $\lambda_{\epsilon} \in \partial \Lambda_{\epsilon}$.

(ii) There exist normalized vectors $u, v \in \mathbb{C}^{n}$ such that

$$
\begin{aligned}
& \sum_{i=1}^{m}\left(A_{i}+\delta A_{i}\right) p_{i}\left(\lambda_{\epsilon}\right) v=0, \\
& u^{*} \sum_{i=1}^{m}\left(A_{i}+\delta A_{i}\right) p_{i}\left(\lambda_{\epsilon}\right)=0,
\end{aligned}
$$

where

$$
\delta A_{i}=-\frac{\overline{p_{i}\left(\lambda_{\epsilon}\right)}}{w_{i}\left|p_{i}\left(\lambda_{\epsilon}\right)\right|} u v^{*} \epsilon, \quad 1 \leq i \leq m
$$

The following relations hold.

1. (i) implies (ii). Moreover, $u$ and $v$ in (ii) can be chosen as left and right singular vectors corresponding to

$$
\sigma_{n}\left(\sum_{i=1}^{m} A_{i} p_{i}\left(\lambda_{\epsilon}\right)\right)=\epsilon\left(\sum_{i=1}^{m} \frac{\left|p_{i}\left(\lambda_{\epsilon}\right)\right|}{w_{i}}\right)
$$

2. If (ii) holds, then there exists an integer $k \in\{1, \ldots n\}$ such that

$$
\sigma_{k}\left(\sum_{i=1}^{m} A_{i} p_{i}\left(\lambda_{\epsilon}\right)\right)=\epsilon\left(\sum_{i=1}^{m} \frac{\left|p_{i}\left(\lambda_{\epsilon}\right)\right|}{w_{i}}\right)
$$

with corresponding right singular vector $v$ and left singular vector $u$. If, in addition, $k=n$ in (3.2), then $\lambda_{\epsilon} \in \partial \Lambda_{\epsilon}$. 
Proof. We start by proving 1 . Since $\lambda \in \partial \Lambda_{\epsilon}$ we have, by Theorem 1 ,

$$
\sigma_{n}\left(\sum_{i=1}^{m} A_{i} p_{i}\left(\lambda_{\epsilon}\right)\right)=\epsilon\left\|w\left(\lambda_{\epsilon}\right)\right\|_{1} .
$$

Let $(u, v)$ be corresponding (normalized) right and left singular vectors. From (3.3) we get

$$
\left(\sum_{i=1}^{m} A_{i} p_{i}\left(\lambda_{\epsilon}\right)\right) v=\epsilon\left\|w\left(\lambda_{\epsilon}\right)\right\|_{1} u, \quad u^{*}\left(\sum_{i=1}^{m} A_{i} p_{i}\left(\lambda_{\epsilon}\right)\right)=\epsilon\left\|w\left(\lambda_{\epsilon}\right)\right\|_{1} v^{*}
$$

By using (1.7) and by the property $u^{*} u=v^{*} v=1$, these equation can be rewritten as

$$
\begin{aligned}
\left(\sum_{i=1}^{m}\left(A_{i}-\epsilon \frac{\left|p_{i}\left(\lambda_{\epsilon}\right)\right|}{w_{i} p_{i}\left(\lambda_{\epsilon}\right)} u v^{*}\right) p_{i}\left(\lambda_{\epsilon}\right)\right) v & =0, \\
u^{*}\left(\sum_{i=1}^{m}\left(A_{i}-\epsilon \frac{\left|p_{i}\left(\lambda_{\epsilon}\right)\right|}{w_{i} p_{i}\left(\lambda_{\epsilon}\right)} u v^{*}\right) p_{i}\left(\lambda_{\epsilon}\right)\right) & =0 .
\end{aligned}
$$

This proves the assertion.

Next we prove 2.; since (ii) can be reformulated as

$$
\left(\sum_{i=1}^{m} A_{i} p_{i}\left(\lambda_{\epsilon}\right)\right) v=\epsilon\left\|w\left(\lambda_{\epsilon}\right)\right\|_{1} u, \quad u^{*}\left(\sum_{i=1}^{m} A_{i} p_{i}\left(\lambda_{\epsilon}\right)\right)=\epsilon\left\|w\left(\lambda_{\epsilon}\right)\right\|_{1} v^{*}
$$

the result follows directly.

The following proposition addresses a property of rightmost points.

Proposition 3.2. Assume that $\lambda_{\epsilon} \in \partial \Lambda_{\epsilon}$. Let $(u, v)$ be a pair of (normalized) left and right singular vectors corresponding to

$$
\sigma_{n}\left(\sum_{i=1}^{m} A_{i} p_{i}\left(\lambda_{\epsilon}\right)\right)
$$

Assume further that $p_{i}\left(\lambda_{\epsilon}\right) \neq 0, i \in\{1, \ldots, m\}$, whenever $w_{i}$ is finite. If $\lambda_{\epsilon}$ is a local maximizer of the optimization problem

$$
\max \left\{\Re(\lambda): \lambda \in \Lambda_{\epsilon}\right\},
$$

then the (first order optimality) conditions

$$
\begin{aligned}
& \Im\left(u^{*}\left(\sum_{i=1}^{m} A_{i} p_{i}^{\prime}\left(\lambda_{\epsilon}\right)\right) v\right)-\epsilon \sum_{i=1}^{m} \frac{\Im\left(\overline{p_{i}\left(\lambda_{\epsilon}\right)} p_{i}^{\prime}\left(\lambda_{\epsilon}\right)\right)}{w_{i}\left|p_{i}\left(\lambda_{\epsilon}\right)\right|}=0, \\
& \Re\left(u^{*}\left(\sum_{i=1}^{m} A_{i} p_{i}^{\prime}\left(\lambda_{\epsilon}\right)\right) v\right)-\epsilon \sum_{i=1}^{m} \frac{\Re\left(\overline{p_{i}\left(\lambda_{\epsilon}\right)} p_{i}^{\prime}\left(\lambda_{\epsilon}\right)\right)}{w_{i}\left|p_{i}\left(\lambda_{\epsilon}\right)\right|}>0,
\end{aligned}
$$

hold. Moreover, if $\lambda_{\epsilon}$ is a global optimizer then it is a rightmost eigenvalue of

$$
\left(\sum_{i=1}^{m}\left(A_{i}-\frac{\overline{p_{i}\left(\lambda_{\epsilon}\right)}}{w_{i}\left|p_{i}\left(\lambda_{\epsilon}\right)\right|} u v^{*} \epsilon\right) p_{i}(\lambda)\right) v=0 .
$$


Proof. Letting $\lambda=a+\mathrm{i} b$, with $a, b \in \mathbb{R}$, Problem (3.4) can be rewritten as

$$
\max _{a, b \in \mathbb{R}} a \quad \text { subject to } \quad \sigma_{n}\left(\sum_{i=1}^{m} A_{i} p_{i}(a+\mathrm{i} b)\right)-\epsilon\|w(a+\mathrm{i} b)\|_{1} \leq 0 .
$$

The fact that $\lambda_{\epsilon}:=a_{\epsilon}+\mathrm{i} b_{\epsilon}$ is a maximizer implies that the constraint is active at the maximum point. Since $p_{i}\left(\lambda_{\epsilon}\right) \neq 0$ whenever $w_{i}$ is finite, the function in the left hand side of (3.8) is differentiable in the neighborhood of $\left(a_{\epsilon}, b_{\epsilon}\right)$. Therefore, there exists a strictly positive Lagrange multiplier $\mu$ such that

$$
0=\left.\frac{\partial \mathcal{L}(a, b, \mu)}{\partial a}\right|_{(a, b)=\left(a_{\epsilon}, b_{\epsilon}\right)}=\left.\frac{\partial \mathcal{L}(a, b, \mu)}{\partial b}\right|_{(a, b)=\left(a_{\epsilon}, b_{\epsilon}\right)},
$$

where

$$
\mathcal{L}(a, b, \mu)=a-\mu\left(\sigma_{n}\left(\sum_{i=1}^{m} A_{i} p_{i}(a+\mathrm{i} b)\right)-\epsilon\|w(a+\mathrm{i} b)\|_{1}\right),
$$

see [20]. From (3.9) we get

$$
\begin{aligned}
& \left.\frac{\partial}{\partial a}\left(\sigma_{n}\left(\sum_{i=1}^{m} A_{i} p_{i}(a+\mathrm{i} b)\right)-\epsilon\|w(a+\mathrm{i} b)\|_{1}\right)\right|_{(a, b)=\left(a_{\epsilon}, b_{\epsilon}\right)}>0, \\
& \left.\frac{\partial}{\partial b}\left(\sigma_{n}\left(\sum_{i=1}^{m} A_{i} p_{i}(a+\mathrm{i} b)\right)-\epsilon\|w(a+\mathrm{i} b)\|_{1}\right)\right|_{(a, b)=\left(a_{\epsilon}, b_{\epsilon}\right)}=0 .
\end{aligned}
$$

Conditions (3.5)-(3.6) follow by noting that

$$
\begin{aligned}
& \frac{\partial}{\partial a}\left(\sigma_{n}\left(\sum_{i=1}^{m} A_{i} p_{i}(a+\mathrm{i} b)\right)\right)=\Re\left(u^{*}\left(\sum_{i=1}^{m} A_{i} p_{i}^{\prime}(a+\mathrm{i} b)\right) v\right), \\
& \frac{\partial}{\partial b}\left(\sigma_{n}\left(\sum_{i=1}^{m} A_{i} p_{i}(a+\mathrm{i} b)\right)\right)=-\Im\left(u^{*}\left(\sum_{i=1}^{m} A_{i} p_{i}^{\prime}(a+\mathrm{i} b)\right) v\right),
\end{aligned}
$$

with $(u, v)$ a pair of normalized left and right singular vectors corresponding to

$$
\sigma_{n}\left(\sum_{i=1}^{m} A_{i} p_{i}(a+\mathrm{i} b)\right)
$$

and by exploiting

$$
\begin{aligned}
\frac{\partial}{\partial a}\|w(a+\mathrm{i} b)\|_{1} & =\sum_{i=1}^{m} \frac{\Re\left(\overline{p_{i}(a+\mathrm{i} b)} p_{i}^{\prime}(a+\mathrm{i} b)\right)}{w_{i}\left|p_{i}(a+\mathrm{i} b)\right|}, \\
\frac{\partial}{\partial b}\|w(a+\mathrm{i} b)\|_{1} & =-\sum_{i=1}^{m} \frac{\Im\left(\overline{p_{i}(a+\mathrm{i} b)} p_{i}^{\prime}(a+\mathrm{i} b)\right)}{w_{i}\left|p_{i}(a+\mathrm{i} b)\right|}
\end{aligned}
$$

for all $a, b \in \mathbb{R}$ satisfying $p_{i}(a+\mathrm{i} b) \neq 0$ whenever $w_{i}$ is finite.

From Proposition 3.1 it follows that $\lambda_{\epsilon}$ is an eigenvalue of (3.7). Since the perturbations in (3.7) have size $\epsilon$ and $\lambda_{\epsilon}$ maximizes (3.4) it must correspond to a rightmost eigenvalue. 
3.2. Algorithm. Define $\lambda_{\epsilon}$ as a globally rightmost point of $\Lambda_{\epsilon}$, i.e.

$$
\Re\left(\lambda_{\epsilon}\right)=\alpha_{\epsilon},
$$

and let $\left(u_{\epsilon}, v_{\epsilon}\right)$ be a pair of (left, right) singular vectors corresponding to

$$
\sigma_{n}\left(\sum_{i=1}^{m} A_{i} p_{i}\left(\lambda_{\epsilon}\right)\right)
$$

uniquely defined by the normalization constraints

$$
u_{\epsilon}^{*} u_{\epsilon}=v_{\epsilon}^{*} v_{\epsilon}=1, c^{*} u_{\epsilon}>0,
$$

where $c \in \mathbb{C}^{n \times 1}$. By Propositions 3.1 and 3.2 the triple $\left(\lambda_{\epsilon}, u_{\epsilon}, v_{\epsilon}\right)$ satisfies the equations

$$
\begin{array}{r}
\left(\sum_{i=1}^{m}\left(A_{i}-\frac{\overline{p_{i}(\lambda)}}{w_{i}\left|p_{i}(\lambda)\right|} u v^{*} \epsilon\right) p_{i}(\lambda)\right) v=0, \\
u^{*}\left(\sum_{i=1}^{m}\left(A_{i}-\frac{\overline{p_{i}(\lambda)}}{w_{i}\left|p_{i}(\lambda)\right|} u v^{*} \epsilon\right) p_{i}(\lambda)\right)=0, \\
u^{*} u=v^{*} v=1, c^{*} u>0, \\
\Im\left(u^{*}\left(\sum_{i=1}^{m} A_{i} p_{i}^{\prime}(\lambda)\right) v\right)-\epsilon \sum_{i=1}^{m} \frac{\Im\left(\overline{p_{i}(\lambda)} p_{i}^{\prime}(\lambda)\right)}{w_{i}\left|p_{i}(\lambda)\right|}=0, \\
\Re\left(u^{*}\left(\sum_{i=1}^{m} A_{i} p_{i}^{\prime}(\lambda)\right) v\right)-\epsilon \sum_{i=1}^{m} \frac{\Re\left(\overline{p_{i}(\lambda} p_{i}^{\prime}(\lambda)\right)}{w_{i}\left|p_{i}(\lambda)\right|}>0 .
\end{array}
$$

Inspired by the algorithm in [11] for the linear eigenvalue problem, conditions (3.10)(3.14), along with the second assertion of Proposition 3.2, can be turned into a fixedpoint iteration, which is sketched in Algorithm 1.

Algorithm 1 (fixed-point iteration).

Initialize $\left(\lambda_{0}, u_{0}, v_{0}\right)$ and repeat for $k=1,2, \ldots$

1. Compute $\lambda_{k}$ as the rightmost eigenvalue of

$$
\left(\sum_{i=1}^{m}\left(A_{i}+\delta A_{i}\right) p_{i}(\lambda)\right) v=0, \quad \delta A_{i}=-\frac{\overline{p_{i}\left(\lambda_{k-1}\right)}}{w_{i}\left|p_{i}\left(\lambda_{k-1}\right)\right|} u_{k-1} v_{k-1}^{*} \epsilon, \quad 1 \leq i \leq m .
$$

2. Define $u_{k}$ and $v_{k}$ as the left and right eigenvectors associated with $\lambda_{k}$, which are scaled such that

$$
\begin{aligned}
& u_{k}^{*} u_{k}=v_{k}^{*} v_{k}=1, c^{*} u_{k}>0, \\
& \Im\left(u_{k}^{*}\left(\sum_{i=1}^{m} A_{i} p_{i}^{\prime}\left(\lambda_{k}\right)\right) v_{k}\right)-\epsilon \sum_{i=1}^{m} \frac{\Im\left(\overline{p_{i}\left(\lambda_{k}\right)} p_{i}^{\prime}\left(\lambda_{k}\right)\right)}{w_{i}\left|p_{i}\left(\lambda_{k}\right)\right|}=0, \\
& \Re\left(u_{k}^{*}\left(\sum_{i=1}^{m} A_{i} p_{i}^{\prime}\left(\lambda_{k}\right)\right) v_{k}\right)-\epsilon \sum_{i=1}^{m} \frac{\Re\left(\overline{p_{i}\left(\lambda_{k}\right)} p_{i}^{\prime}\left(\lambda_{k}\right)\right)}{w_{i}\left|p_{i}\left(\lambda_{k}\right)\right|}>0 .
\end{aligned}
$$


By construction, constraints (3.16)-(3.18) in Algorithm 1 can always be satisfied (i.e., a new iterate is well defined) if the previous iterate $\left(\lambda_{k-1}, u_{k-1}, v_{k-1}\right)$ lies in the neighborhood of the fixed point $\left(\lambda_{\epsilon}, u_{\epsilon}, v_{\epsilon}\right)$. However, for an arbitrary value of $\left(\lambda_{k-1}, u_{k-1}, v_{k-1}\right)$ the constraints (3.16)-(3.18) may be too restrictive. First, if for a given value of $\left(\lambda_{k-1}, u_{k-1}, v_{k-1}\right)$ and corresponding rightmost eigenvalue $\lambda_{k}$ of (3.15) a pair of normalized eigenvector $(u, v)$ is such that

$$
\left|u^{*}\left(\sum_{i=1}^{m} A_{i} p_{i}^{\prime}\left(\lambda_{k}\right)\right) v\right|<\epsilon\left|\sum_{i=1}^{m} \frac{\Im\left(\overline{p_{i}\left(\lambda_{k}\right)} p_{i}^{\prime}\left(\lambda_{k}\right)\right)}{w_{i}\left|p_{i}\left(\lambda_{k}\right)\right|}\right|,
$$

then (3.16)-(3.18) can never be satisfied simultaneously. In order to define the iteration also in this case, we require that (3.17) is satisfied only in least squares sense. Second, there are in general two sets of eigenvectors $\left(u_{k}, v_{k}\right)$ satisfying the normalizing equations (3.16)-(3.17): one for which $\Re\left(u_{k}^{*}\left(\sum_{i=1}^{m} A_{i} p_{i}^{\prime}\left(\lambda_{k}\right)\right) v_{k}\right)$ is positive and one for which it is negative. Although we have never encountered this situation in the experiments, it might occur that both solutions satisfy (3.18) or that none of the solutions satisfies (3.18). In these cases, both solutions are initially considered in the next iteration, but after the computation of the spectral abscissa, $\Re\left(\lambda_{k+1}\right)$, the one that leads to the smallest value is discarded. This brings us to the following algorithm.

ALGORITHM 2 (computation of the pseudospectral abscissa).

1. Set $u_{0}=v_{0}=0$.

2. Repeat for $k=1,2, \ldots$ (until convergence):

Let $\lambda_{k}$ be the rightmost eigenvalue ${ }^{1}$ of

$$
\left(\sum_{i=1}^{m}\left(A_{i}+\delta A_{i}\right) p_{i}(\lambda)\right) v=0, \quad \delta A_{i}=-\frac{\overline{p_{i}\left(\lambda_{k-1}\right)}}{w_{i}\left|p_{i}\left(\lambda_{k-1}\right)\right|} u_{k-1} v_{k-1}^{*} \epsilon, \quad 1 \leq i \leq m .
$$

Define $u_{k}$ and $v_{k}$ as the left and right eigenvectors associated with $\lambda_{k}$, which are scaled such that

$$
\left\|u_{k}\right\|_{2}=\left\|v_{k}\right\|_{2}=1, a^{*} u_{k}>0
$$

the value of

$$
\left|\Im\left(u_{k}^{*}\left(\sum_{i=1}^{m} A_{i} p_{i}^{\prime}\left(\lambda_{k}\right)\right) v_{k}\right)-\epsilon \sum_{i=1}^{m} \frac{\Im\left(\overline{p_{i}\left(\lambda_{k}\right)} p_{i}^{\prime}\left(\lambda_{k}\right)\right)}{w_{i}\left|p_{i}\left(\lambda_{k}\right)\right|}\right|
$$

is minimal and

$$
\Re\left(u_{k}^{*}\left(\sum_{i=1}^{m} A_{i} p_{i}^{\prime}\left(\lambda_{k}\right)\right) v_{k}\right)-\epsilon \sum_{i=1}^{m} \frac{\Re\left(\overline{p_{i}\left(\lambda_{k}\right)} p_{i}^{\prime}\left(\lambda_{k}\right)\right)}{w_{i}\left|p_{i}\left(\lambda_{k}\right)\right|} \geq 0 .
$$

If a pair $\left(u_{k}, v_{k}\right)$ satisfying the conditions (3.20)-(3.22) does not exist or is not unique, then select the eigenvector pair satisfying (3.20)-(3.21) for which

$$
\alpha\left(\left(\sum_{i=1}^{m}\left(A_{i}+\delta A_{i}\right) p_{i}(\lambda)\right)\right), \quad \delta A_{i}=-\frac{\overline{p_{i}\left(\lambda_{k}\right)}}{w_{i}\left|p_{i}\left(\lambda_{k}\right)\right|} u_{k} v_{k}^{*} \epsilon, \quad 1 \leq i \leq m,
$$

is maximal.

\footnotetext{
${ }^{1}$ If the rightmost eigenvalue is not unique, select the one closest to $\lambda_{k-1}$.
} 
By construction the iterates generated by Algorithm 2 satisfy the following property.

Proposition 3.3. Algorithm 2 generates a sequence $\left\{\Re\left(\lambda_{k}\right)\right\}_{k \geq 0}$ of lower bounds on the pseudospectral abscissa.

Proof. It is clear that $\Re\left(\lambda_{1}\right) \leq \alpha_{\epsilon}$. For all $k \geq 2, \lambda_{k}$ is a solution of the perturbed eigenvalue problem (3.19), where the perturbations satisfy

$$
\left\|\left(\delta A_{1}, \ldots, \delta A_{m}\right)\right\|_{\text {glob }}=\epsilon
$$

implying $\Re\left(\lambda_{k}\right) \leq \alpha_{\epsilon}$.

4. Convergence analysis. In this section we show that under mild conditions both Algorithm 1 and Algorithm 2 have a unique fixed point for small $\epsilon$, which is attractive and corresponds to a globally rightmost point of the pseudospectrum. The convergence rate as a function of $\epsilon$ is also discussed.

4.1. Characterization of fixed points. In $\S 3.2$ we have shown by construction that a rightmost point of the pseudospectrum defines a fixed point of the iteration in Algorithm 1. The following lemma presents a converse result.

Lemma 4.1. Assume that $\left(\lambda_{\epsilon}, u_{\epsilon}, v_{\epsilon}\right)$ is a fixed point of the iteration in Algorithm 1. Then there exists a number $\ell \in\{1, \ldots, n\}$ such that

$$
\sigma_{\ell}\left(\sum_{i=1}^{m} A_{i} p_{i}\left(\lambda_{\epsilon}\right)\right)=\epsilon\left(\sum_{i=1}^{m} \frac{\left|p_{i}\left(\lambda_{\epsilon}\right)\right|}{w_{i}}\right) .
$$

Moreover, the outward-pointing normal vector to the set

$$
\left\{\lambda \in \mathbb{C}: \sigma_{\ell}\left(\sum_{i=1}^{m} A_{i} p_{i}(\lambda)\right)-\epsilon\left(\sum_{i=1}^{m} \frac{\left|p_{i}(\lambda)\right|}{w_{i}}\right) \leq 0\right\}
$$

at $\lambda=\lambda_{\epsilon}$ lies in the direction of the positive real axis.

Proof. The conditions for a fixed point imply the first assertion, see Proposition 3.1. The outward-pointing normal has direction

$$
\left(\frac{\partial f}{\partial a}\left(\Re\left(\lambda_{\epsilon}\right), \Im\left(\lambda_{\epsilon}\right)\right), \frac{\partial f}{\partial b}\left(\Re\left(\lambda_{\epsilon}\right), \Im\left(\lambda_{\epsilon}\right)\right)\right),
$$

where

$$
f(a, b):=\sigma_{\ell}\left(\sum_{i=1}^{m} A_{i} p_{i}(a+\mathrm{i} b)\right)-\epsilon\left(\sum_{i=1}^{m} \frac{\left|p_{i}(a+\mathrm{i} b)\right|}{w_{i}}\right) .
$$

The expressions

$$
\begin{aligned}
& \frac{\partial f}{\partial a}\left(\Re\left(\lambda_{\epsilon}\right), \Im\left(\lambda_{\epsilon}\right)\right)=\Re\left(u_{\epsilon}^{*}\left(\sum_{i=1}^{m} A_{i} p_{i}^{\prime}\left(\lambda_{\epsilon}\right)\right) v_{\epsilon}\right)-\epsilon \sum_{i=1}^{m} \frac{\Re\left(\overline{p_{i}\left(\lambda_{\epsilon}\right)} p_{i}^{\prime}\left(\lambda_{\epsilon}\right)\right)}{w_{i}\left|p_{i}\left(\lambda_{\epsilon}\right)\right|}, \\
& \frac{\partial f}{\partial b}\left(\Re\left(\lambda_{\epsilon}\right), \Im\left(\lambda_{\epsilon}\right)\right)=\Im\left(u_{\epsilon}^{*}\left(\sum_{i=1}^{m} A_{i} p_{i}^{\prime}\left(\lambda_{f}\right)\right) v_{\epsilon}\right)-\epsilon \sum_{i=1}^{m} \frac{\Im\left(\overline{p_{i}\left(\lambda_{\epsilon}\right)} p_{i}^{\prime}\left(\lambda_{\epsilon}\right)\right)}{w_{i}\left|p_{i}\left(\lambda_{\epsilon}\right)\right|},
\end{aligned}
$$

along with the condition for a fixed point lead to the second assertion. 
In order to strengthen this result for small values of $\epsilon$ we need another technical lemma, which describes the shape of the pseudospectral components.

Lemma 4.2. Assume $\hat{\lambda}$ is an eigenvalue of (1.1) with multiplicity one such that

$$
\operatorname{det}\left(\sum_{i=1}^{m} A_{i} p_{i}^{\prime}(\hat{\lambda})\right) \neq 0 \text {. }
$$

Then there exists a constant $\gamma>0$ such that

$$
\frac{\sigma_{n}\left(\sum_{i=1}^{m} A_{i} p_{i}(\lambda)\right)}{\|w(\lambda)\|_{1}}=\gamma|\lambda-\hat{\lambda}|+\mathcal{O}\left(|\lambda-\hat{\lambda}|^{2}\right) .
$$

Consequently, if $\epsilon$ tends to zero, then $N_{\epsilon}(\hat{\lambda})$, the component of $\Lambda_{\epsilon}$ containing $\hat{\lambda}$, tends to a disc centered at $\hat{\lambda}$ with radius proportional to $\epsilon$.

Proof. Without loosing generality we assume $\hat{\lambda}=0$. We can now slightly adapt the proof of Theorem 7.4 in [5], addressing pseudospectra of linear eigenvalue problems. First the expansion for the smallest singular value of a linear eigenvalue problem, $\sigma_{n}(A-\lambda I)$, in [5] can be generalized to a pencil, $\sigma_{n}(M+N \lambda)$, where $\operatorname{det}(N) \neq 0$. Second, it is easy to see that expansion remains valid when considering higher order terms, $\sigma_{n}\left(M+N \lambda+\mathcal{O}\left(|\lambda|^{2}\right)\right)$. The lemma follows from

$$
\sum_{i=1}^{m} A_{i} p_{i}(\lambda)=\left(\sum_{i=1}^{m} A_{i} p_{i}(0)\right)+\left(\sum_{i=1}^{m} A_{i} p_{i}^{\prime}(0)\right) \lambda+\mathcal{O}\left(|\lambda|^{2}\right)
$$

and $\|w(\lambda)\|_{1}=\|w(0)\|_{1}+\mathcal{O}(|\lambda|)$.

By combining Lemma 4.1 and Lemma 4.2 we arrive at the following result.

Proposition 4.3. Assume that $\lambda$ is a rightmost eigenvalue of (1.1) with multiplicity one and satisfying (4.2). Assume in addition that the rightmost eigenvalue is unique ${ }^{2}$. Then for sufficiently small $\epsilon$ the iteration map defined by either Algorithm 1 or Algorithm 2 has a unique fixed point

$$
\left(\lambda_{\epsilon}, u_{\epsilon}, v_{\epsilon}\right)
$$

which implies $\Re\left(\lambda_{\epsilon}\right)=\alpha_{\epsilon}$. Moreover, we have

$$
\lim _{\epsilon \rightarrow 0} u_{\epsilon}=u, \lim _{\epsilon \rightarrow 0} v_{\epsilon}=v
$$

where $(u, v)$ is a pair of left and right eigenvectors corresponding to $\lambda$, normalized in such a way that

$$
u^{*}\left(\sum_{i=1}^{m} A_{i} p_{i}^{\prime}(\lambda)\right) v>0
$$

Proof. We first consider the fixed points of Algorithm 1. By the first assertion of Proposition 4.1 all fixed points must belong to $\partial \Lambda_{\epsilon}$. By Lemma 4.2 and the fact that the number of eigenvalues in any right half plane is finite, a fixed point must belong to $N_{\epsilon}(\lambda)$ for sufficiently small $\epsilon$.

\footnotetext{
${ }^{2}$ If the spectrum is symmetric w.r.t. the real axis, then only the eigenvalues in the upper half plane are considered
} 
For small $\epsilon$ condition (4.1) cannot be satisfied for $\ell \neq n$ since

$$
\sigma_{n-1}\left(\sum_{i=1}^{m} A_{i} p_{i}(\lambda)\right)>0 .
$$

Moreover, the circular shape of $N_{\epsilon}(\lambda)$, described by Lemma 4.2, is such is that there is a unique point in $N_{\epsilon}(\lambda)$, satisfying the properties described by Lemma 4.1, namely the rightmost point.

The continuity of the function $\epsilon \mapsto \lambda_{\epsilon}$ for small $\epsilon$ implies the continuity of the functions $\epsilon \mapsto u_{\epsilon}$ and $\epsilon \mapsto v_{\epsilon}$. From

$$
\begin{aligned}
& \Im\left(u_{\epsilon}^{*}\left(\sum_{i=1}^{m} A_{i} p_{i}^{\prime}\left(\lambda_{\epsilon}\right)\right) v_{\epsilon}\right)-\epsilon \sum_{i=1}^{m} \frac{\Im\left(\overline{p_{i}\left(\lambda_{\epsilon}\right)} p_{i}^{\prime}\left(\lambda_{\epsilon}\right)\right)}{w_{i}\left|p_{i}\left(\lambda_{\epsilon}\right)\right|}=0, \\
& \Re\left(u_{\epsilon}^{*}\left(\sum_{i=1}^{m} A_{i} p_{i}^{\prime}\left(\lambda_{\epsilon}\right)\right) v_{\epsilon}\right)-\epsilon \sum_{i=1}^{m} \frac{\Re\left(\overline{p_{i}\left(\lambda_{\epsilon}\right)} p_{i}^{\prime}\left(\lambda_{\epsilon}\right)\right)}{w_{i}\left|p_{i}\left(\lambda_{\epsilon}\right)\right|}>0,
\end{aligned}
$$

a limit argument yields

$$
u^{*}\left(\sum_{i=1}^{m} A_{i} p_{i}^{\prime}(\lambda)\right) v \geq 0 .
$$

Because $\lambda$ is a simple eigenvalue we have $u^{*}\left(\sum_{i=1}^{m} A_{i} p_{i}^{\prime}(\lambda)\right) v \neq 0$, as the equality would imply the existence of a Jordan chain of length at least two [13]. Expression (4.6) follows.

The above analysis carries over to Algorithm 2, since for small values of $\epsilon$ all iterates lie in the vicinity of (4.4), where Algorithm 2 reduces to Algorithm 1.

4.2. Convergence of the fixed-point iteration. We analyze the convergence of Algorithm 2 around the fixed point (4.4) for small values of $\epsilon$. Note that under the assumptions of Proposition 4.3 Algorithm 2 reduces to Algorithm 1 if $\epsilon$ is small.

We start with the observation that the dependence of the new iterate on the previous one is via terms containing $\epsilon$. Therefore, setting

$$
x_{k}:=\left[\Re\left(\lambda_{k}\right) \Im\left(\lambda_{k}\right) \Re\left(u_{k}\right)^{T} \Im\left(u_{k}\right)^{T} \Re\left(v_{k}\right)^{T} \Im\left(v_{k}\right)^{T}\right]^{T},
$$

the iteration map defined by Algorithm 1 / Algorithm 2 can be written as a real valued mapping of the form

$$
x_{k}=F\left(\epsilon G\left(x_{k-1}\right), \epsilon\right)
$$

where

$$
G\left(x_{k-1}\right)=\operatorname{vec}\left[\begin{array}{c}
\Re\left(\frac{\overline{p_{1}\left(\lambda_{k-1}\right)}}{w_{1} p_{1}\left(\lambda_{k-1}\right)} u_{k-1} v_{k-1}^{*}\right) \\
\Im\left(\frac{\overline{p_{1}\left(\lambda_{k-1}\right)}}{w_{1} p_{1}\left(\lambda_{k-1}\right)} u_{k-1} v_{k-1}^{*}\right) \\
\vdots \\
\Re\left(\frac{\frac{p_{m}\left(\lambda_{k-1}\right)}{w_{m} p_{m}\left(\lambda_{k-1}\right)}}{u_{k-1}} v_{k-1}^{*}\right) \\
\Im\left(\frac{\frac{p_{m}\left(\lambda_{k-1}\right)}{w_{m} p_{m}\left(\lambda_{k-1}\right)}}{u_{k-1}} v_{k-1}^{*}\right)
\end{array}\right] .
$$


Under the assumptions of Proposition 4.3 partial derivatives of $G$ and $F$ exist, the latter following from the differentiability of an isolated eigenvalue with respect to parameters on which the characteristic matrix affinity depends. Moreover, the point

$$
x_{\epsilon}:=\left[\Re\left(\lambda_{\epsilon}\right) \Im\left(\lambda_{\epsilon}\right) \Re\left(u_{\epsilon}\right)^{T} \Im\left(u_{\epsilon}\right)^{T} \Re\left(v_{\epsilon}\right)^{T} \Im\left(v_{\epsilon}\right)^{T}\right]^{T}
$$

is an isolated fixed point of (4.7). The Jacobian of $F(\epsilon G(\cdot), \epsilon)$ at $x_{\epsilon}$ is given by

$$
J_{\epsilon}:=\left.\epsilon \frac{d G}{d x}\left(x_{\epsilon}\right) \frac{\partial F(u, \epsilon)}{\partial u}\right|_{u=\epsilon G\left(x_{\epsilon}\right)} .
$$

It has the following properties.

THEOREM 4.4. Assume that the conditions of Proposition 4.3 hold.

1. For sufficiently small $\epsilon$ we have

$$
\rho\left(J_{\epsilon}\right)<1
$$

where $J_{\epsilon}$ is the Jacobian (4.8) of the iteration map defined by Algorithm 2.

2. We have $\lim _{\epsilon \rightarrow 0^{+}} \rho\left(J_{\epsilon}\right)=0$.

3. The first row of $J_{\epsilon}$ is identically zero.

Proof. It is clear that $\left\|\frac{d G}{d x}\left(x_{\epsilon}\right)\right\|_{2}$ can be uniformly bounded over $\epsilon \in(0, \bar{\epsilon})$, where $\bar{\epsilon}$ is sufficiently small. This is also the case for

$$
\left\|\left.\frac{\partial F(u, \epsilon)}{\partial u}\right|_{u=G\left(x_{\epsilon}\right)}\right\|_{2} .
$$

Indeed, partial derivatives of $F$ with respect to elements of $u$ concern the sensitivity (derivative) of an isolated eigenvalue with respect to a parameter on which the characteristic matrix depends. All derivatives can be expressed as fractions, whose numerator is given by

$$
g(\epsilon):=u_{\epsilon}^{*}\left(\sum_{i=1}^{m}\left(A_{i}-\frac{\overline{p_{i}\left(\lambda_{\epsilon}\right)}}{w_{i}\left|p_{i}\left(\lambda_{\epsilon}\right)\right|} u_{\epsilon} v_{\epsilon}^{*} \epsilon\right) p_{i}^{\prime}\left(\lambda_{\epsilon}\right)\right) v_{\epsilon} .
$$

We have (for $\lambda$ the rightmost eigenvalue and $u, v$ associated eigenvectors)

$$
\lim _{\epsilon \rightarrow 0+} g(\epsilon)=u^{*}\left(\sum_{i=1}^{m} A_{i} p_{i}^{\prime}(\lambda)\right) v
$$

which is strictly positive by Proposition 4.3. This implies that (4.10) can be bounded by a constant independent of $\epsilon$. Assertion 1. and Assertion 2. follow from the structure (4.8) of $J_{\epsilon}$.

For fixed $\epsilon$ the iteration, sufficiently close to the fixed point, is written as

$$
\Delta x_{k}=J(\epsilon) \Delta x_{k-1}+\mathcal{O}\left(\left\|\Delta x_{k-1}\right\|^{2}\right),
$$

where $\Delta x_{k}=x_{k}-x_{\epsilon}$. From the property that

$$
\left[\begin{array}{llll}
1 & 0 & \cdots & 0
\end{array}\right] \Delta x_{k} \leq 0
$$

for $k \geq 1$ (see Proposition 3.3), we conclude that Assertion 3. must hold. 
The practical meaning of Theorem 4.4 can be summarized as follows. Expression (4.9) implies that the fixed point is attractive. By Staement 2. in Proposition 4.4, the rate of convergence tends to zero as $\epsilon$ tends to zero. The third assertion allows to explain qualitatively the behavior of the algorithm in the neighborhood of the fixed point. First, if $\left[\begin{array}{lllll}0 & 1 & 0 & \cdots & 0\end{array}\right] e_{1} \neq 0$, for some eigenvector $e_{1}$ corresponding to a dominant eigenvalue of $J_{\epsilon}$, then we generically obtain

$$
\lim _{k \rightarrow \infty} \frac{\Re\left(\lambda_{k}\right)-\Re\left(\lambda_{\epsilon}\right)}{\Im\left(\lambda_{k}\right)-\Im\left(\lambda_{\epsilon}\right)}=0,
$$

i.e., the rightmost point of the pseudospectrum is approached along the boundary. This behavior is frequently observed in experiments. An exception is formed by the case where $\lambda_{k} \in \mathbb{R}$ and $u_{k}, v_{k} \in \mathbb{R}^{n}$ for all values of $k$, which may occur if all matrices $A_{i}$ are real. Second, the third assertion of the proposition implies the expansion

$$
\Re\left(\lambda_{k}\right)-\Re\left(\lambda_{\epsilon}\right)=\Delta x_{k-1}^{T} H_{\epsilon} \Delta x_{k-1}+\mathcal{O}\left(\left\|\Delta x_{k-1}\right\|_{2}^{3}\right),
$$

where $H_{\epsilon}$ is the Hessian of $\left[\begin{array}{lll}1 & 0 & \cdots\end{array}\right] F(\epsilon G(x), \epsilon)$ at $x=x_{\epsilon}$. If the dominant eigenvalue of $J_{\epsilon}$ is unique, real, and its corresponding eigenvector $e_{1}$ satisfies $e_{1}^{T} H_{\epsilon} e_{1} \neq 0$ then we have for almost all values for $\Delta x_{0}$,

$$
\lim _{k \rightarrow \infty} \frac{\left|\Re\left(\lambda_{k}\right)-\Re\left(\lambda_{\epsilon}\right)\right|}{\left|\Re\left(\lambda_{k-1}\right)-\Re\left(\lambda_{\epsilon}\right)\right|}=\rho\left(J_{\epsilon}\right)^{2},
$$

i.e., the convergence rate of the spectral abscissa is the square of the convergence rated for the whole iteration. We conclude the section with an illustration of Theorem 4.4.

Example 1. Consider the delay eigenvalue problem

$$
\left(\lambda I-\left(A_{1}+\delta A_{1}\right)-\left(A_{2}+\delta A_{2}\right) e^{-\lambda \tau}\right) v=0
$$

where

$$
A_{1}=\left[\begin{array}{rr}
-5 & 1 \\
2 & -6
\end{array}\right], A_{2}=\left[\begin{array}{rr}
-2 & 1 \\
4 & -1
\end{array}\right], \tau=1,
$$

and the allowable perturbations satisfy

$$
w_{i}\left\|\delta A_{1}\right\|<\epsilon, i=1,2 .
$$

For $\epsilon=0.5$ and weights $\left(w_{1}, w_{2}\right)=(2,2)$ Algorithm 2 converges to a fixed point corresponding to the pseudospectral abscissa $\alpha_{\epsilon}=-0.51816262$. The error between the iterates and the fixed point is displayed in Figure 4.1. The results are consistent with the above conclusions. In particular, the convergence is linear and the convergence rate of the real part of the eigenvalue is twice the convergence rate for the sequence $\left\{\left\|x_{k}-x_{\epsilon}\right\|_{2}\right\}_{k \geq 1}$.

5. Behavior for large values of $\epsilon$ and algorithmic improvements. In Section 4 we proved that Algorithm 2 converges to a fixed point corresponding to the globally rightmost point of the pseudospectrum for sufficiently small values of $\epsilon$. From intensive experiments on a variety of problems we observe that in general the algorithm works very well for large values of $\epsilon$ too. Exceptionally the following problems were observed: i) convergence to an attractor different from a fixed point / an unstable fixed point, and, ii) convergence to a locally but not globally rightmost point of the pseudospectrum contour. In what follows we show how these problems have been solved by improvements of the algorithm. 


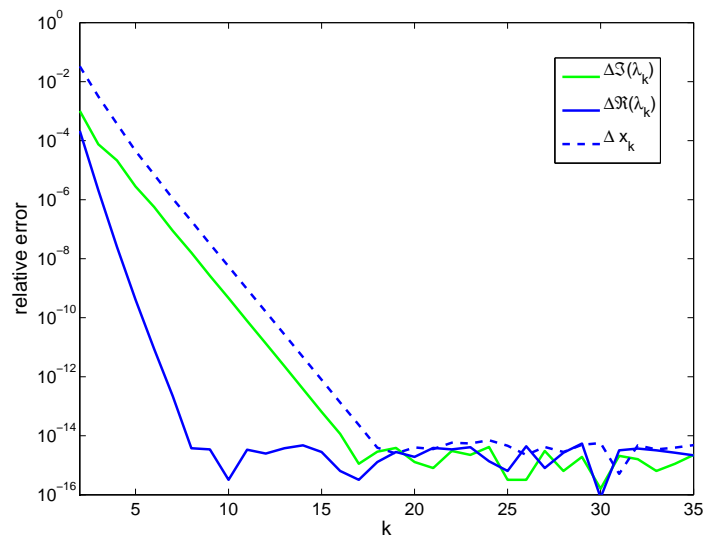

FIG. 4.1. Errors in the iterations of Algorithm 2 applied to Example 1.

5.1. Imposing monotone behavior and practical stability of fixed-points. In rare cases convergence of Algorithm 2 to the fixed point corresponding to the pseudospectral abscissa is not obtained, due to the fact that the initial iterate is included in the attraction region of a different attractor of the nonlinear map We now present an adaptation of the algorithm for which sequence

$$
\left\{\Re\left(\lambda_{k}\right)\right\}_{k \geq 1},
$$

is guaranteed to be increasing, and, as it is bounded from above, converging. Subsequently, we illustrate the benefits of the adapted algorithm.

Consider subsequent iterations of Algorithm 2 and define

$$
P_{i, k}=\frac{\overline{p_{i}\left(\lambda_{k}\right)}}{\left|p_{i}\left(\lambda_{k}\right)\right|}, k \geq 0,1 \leq i \leq m
$$

After iteration step $\ell$, with $\ell \geq 2$, we have that $\lambda_{\ell}$ is a solution of the perturbed eigenvalue problem

$$
\sum_{i=1}^{m}\left(A_{i}+\delta A_{i}^{(\ell)}\right) p_{i}(\lambda)=0
$$

where

$$
\delta A_{i}^{(\ell)}=P_{i, \ell-1} u_{\ell-1} v_{\ell-1}^{*} \epsilon, \quad 1 \leq i \leq m .
$$

Note that the perturbations (5.3) have size $\epsilon$ for $\|\cdot\|_{\text {glob }}$. If a situation occurs where

$$
\Re\left(\lambda_{k}\right)<\Re\left(\lambda_{k-1}\right),
$$

then monotonicity can be enforced by constructing new perturbations of size $\epsilon$ from 2 consecutive iterations, more precisely from (5.3) for $\ell=k-1$ and $\ell=k$, which eventually replace $\delta A_{i}^{(k)}$ and for which the corresponding eigenvalue has real part larger or equal to $\Re\left(\lambda_{k-1}\right)$. 
Let $t \in[0,1]$ and consider the following homotopy:

$$
\delta A_{i}(t)=-\frac{1}{w_{i}} P(t) U(t) V(t)^{*} \epsilon, 1 \leq i \leq m,
$$

where

$$
\begin{aligned}
& P_{i}(t)=\frac{t^{2} P_{i, k-1}+\left(1-t^{2}\right) P_{i, k-2}}{\left|t^{2} P_{i, k-1}+\left(1-t^{2}\right) P_{i, k-2}\right|}, \quad 1 \leq i \leq m \\
& U(t)=\frac{t u_{k-1}+(1-t) u_{k-2}}{\left\|t u_{k-1}+(1-t) u_{k-2}\right\|_{2}}, \quad V(t)=\frac{t v_{k-1}+(1-t) v_{k-2}}{\left\|t v_{k-1}+(1-t) v_{k-2}\right\|_{2}} .
\end{aligned}
$$

For all $t \in[0,1]$ we have

$$
\left\|\left(\delta A_{1}(t), \ldots, \delta A_{m}(t)\right)\right\|_{\text {glob }}=\epsilon .
$$

For $t=0$ the eigenvalue problem

$$
\left(\sum_{i=1}^{m}\left(A_{i}+\delta A_{i}(t)\right) p_{i}(\lambda)\right) v=0
$$

has a solution $\lambda_{k-1}$, with right eigenvector $v_{k-1}$ and left eigenvector $u_{k-1}$. If this eigenvalue is simple, then (5.7) locally defines a continuous function $t \mapsto \lambda(t)$, satisfying $\lambda(0)=\lambda_{k-1}$. A straightforward computation yields

$$
\begin{aligned}
& \dot{\lambda}(0)=\epsilon\left(\sum_{i=1}^{m} \frac{P_{i, k-2} p_{i}\left(\lambda_{k-1}\right)}{w_{i}}\right) \\
& \frac{\left(u_{k-1}^{*} u_{k-2}\left(1-v_{k-2}^{*} v_{k-1} \Re\left(v_{k-2}^{*} v_{k-1}\right)\right)+\left(1-u_{k-1}^{*} u_{k-2} \Re\left(u_{k-2}^{*} u_{k-1}\right)\right) v_{k-2}^{*} v_{k-1}\right)}{u_{k-1}^{*}\left(\sum_{i=1}^{m}\left(A_{i}-\frac{P_{i, k-2}}{w_{i}} u_{k-2} v_{k-2}^{*} \epsilon\right) p_{i}^{\prime}\left(\lambda_{k-1}\right)\right) v_{k-1}}
\end{aligned}
$$

where we note that the sign of (5.8) can be altered by changing the sign of $u_{k-1}$ and $v_{k-1}$, however, such a sign change does not affect the perturbations (5.3), i.e. (5.5) for $t=1$. In other words, the sign change affects the path of the homotopy, but not the initial and final perturbation. Assume now that such a sign change has possibly been done and we are in a situation where

$$
\Re(\dot{\lambda}(0))>0 .
$$

Now we can start with $t=1$ and decrease the value of $t$ (back tracking) until the perturbed eigenvalue problem (5.7) has a solution $\lambda$, satisfying $\Re(\lambda)>\Re\left(\lambda_{k-1}\right)$. This eigenvalue and the corresponding perturbation (5.5)-(5.6) can be used to replace original results of the $k$-th iteration step, namely $\lambda_{k}$ and $\delta A_{i}^{(k)}, 1 \leq i \leq m$.

In the implementation we relax the non-monotonicity condition (5.4) to

$$
\Re\left(\lambda_{k}\right)<\max _{l \leq k-1} \Re\left(\lambda_{l}\right)-\text { tol, }
$$

where tol $>0$ is a small tolerance (default value $10^{-10}$ ). The reason is that if the algorithm has converged, rounding errors may cause small fluctuations of $\left\{\Re\left(\lambda_{k}\right)\right\}$ which would otherwise trigger an inner iteration. In this way we arrive at the following algorithm:

Algorithm 3. (computation of the pseudospectral abscissa, monotone variant) 
1. Set $u_{0}=v_{0}=0$.

2. Repeat for $k=1,2, \ldots$ (until convergence):

Let $\lambda_{k}$ be the rightmost eigenvalue of

$$
\left(\sum_{i=1}^{m}\left(A_{i}+\delta A_{i}\right) p_{i}(\lambda)\right) v=0, \quad \delta A_{i}=-\frac{P_{i, k-1}}{w_{i}} u_{k-1} v_{k-1}^{*} \epsilon, \quad 1 \leq i \leq m .
$$

If $k>2, \Re\left(\lambda_{k}\right)<\max _{l \leq k-1} \Re\left(\lambda_{l}\right)-$ tol and $|\Re(\dot{\lambda}(0))| \neq 0$, (see (5.8)), then If $\Re(\dot{\lambda}(0))<0$, set $u_{k-1}=-u_{k-1}, v_{k-1}=-v_{k-1}$ Set $t=1$.

While $\Re\left(\lambda_{k}\right)<\Re\left(\lambda_{k-1}\right)$,

Set $t=\frac{t}{2}$.

Recompute $\lambda_{k}$ as the rightmost eigenvalue of

$$
\left(\sum_{i=1}^{m}\left(A_{i}+\delta A_{i}\right) p_{i}(\lambda)\right) v=0, \quad \delta A_{i}=-\frac{P_{i}(t)}{w_{i}} U(t) V(t)^{*} \epsilon, \quad 1 \leq i \leq m,
$$

where $U(t), V(t)$ and $P_{i}(t), 1 \leq i \leq m$, are given by (5.6).

End While

End If

Set $u_{k-1}=U(t), v_{k-1}=V(t)$ and $P_{i, k-1}=P_{i}(t), \quad 1 \leq i \leq m$.

Set

$$
P_{i, k}=\frac{\overline{p_{i}\left(\lambda_{k}\right)}}{\left|p_{i}\left(\lambda_{k}\right)\right|}, 1 \leq i \leq m .
$$

Let $u_{k}$ and $v_{k}$ be the left and right eigenvectors associated with $\lambda_{k}$, which are scaled such that

$$
\left\|u_{k}\right\|_{2}=\left\|v_{k}\right\|_{2}=1, a^{*} u_{k}>0
$$

the value of

$$
\left|\Im\left(u_{k}{ }^{*}\left(\sum_{i=1}^{m} A_{i} p_{i}^{\prime}\left(\lambda_{k}\right)\right) v_{k}\right)-\epsilon \sum_{i=1}^{m} \frac{\Im\left(\overline{p_{i}\left(\lambda_{k}\right)} p_{i}^{\prime}\left(\lambda_{k}\right)\right)}{w_{i}\left|p_{i}\left(\lambda_{k}\right)\right|}\right|
$$

is minimal and

$$
\Re\left(u_{k}^{*}\left(\sum_{i=1}^{m} A_{i} p_{i}^{\prime}\left(\lambda_{k}\right)\right) v_{k}\right)-\epsilon \sum_{i=1}^{m} \frac{\Re\left(\overline{p_{i}\left(\lambda_{k}\right)} p_{i}^{\prime}\left(\lambda_{k}\right)\right)}{w_{i}\left|p_{i}\left(\lambda_{k}\right)\right|} \geq 0 .
$$

If an eigenvector pair $\left(u_{k}, v_{k}\right)$ satisfying the conditions (5.10)-(5.12) does not exist or is not unique, then select the eigenvector pair satisfying (5.10)-(5.11) for which

$$
\alpha\left(\left(\sum_{i=1}^{m}\left(A_{i}+\delta A_{i}\right) p_{i}(\lambda)\right)\right), \quad \delta A_{i}=-\frac{P_{i, k}}{w_{i}} u_{k} v_{k}^{*} \epsilon, \quad 1 \leq i \leq m,
$$

is maximal. 

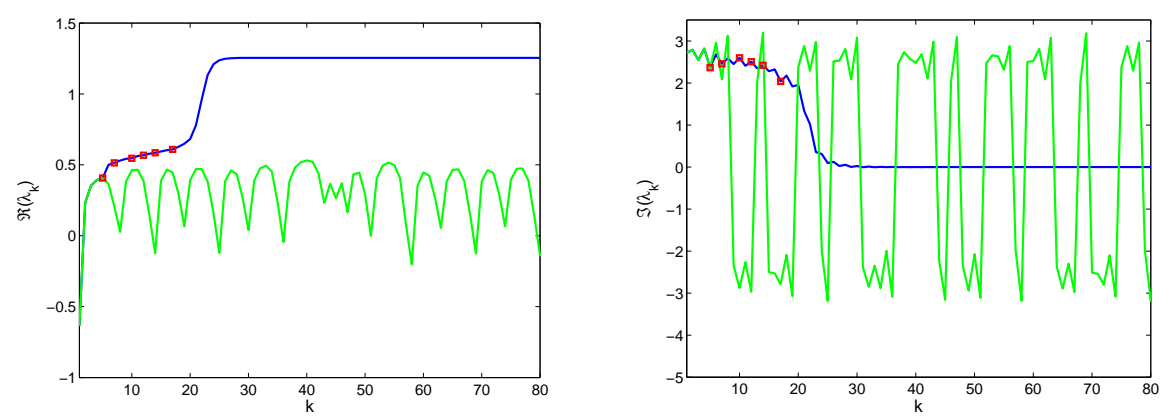

FIG. 5.1. Result of iterations of Algorithm 2 (green curves) and Algorithm 3 (blue curves) when applied to Example 2. Iterations for which (5.9) is satisfied $\left(\right.$ tol $\left.=10^{-10}\right)$ are indicated with red squares.

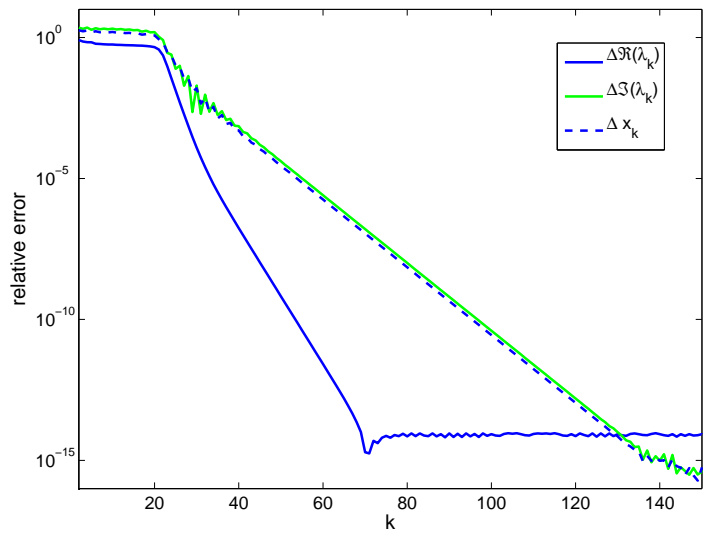

FIG. 5.2. Convergence of Algorithm 3 applied to Example 2.

For any $k \in \mathbb{N}$, Algorithm 3 shares the desired property with Algorithm 2 that at the end of the k-th iteration, $\lambda_{k}$ is an eigenvalue of (5.2) corresponding to the $\epsilon$-sized perturbations (5.3) but, if monotonicity needs to be forced at Iteration $k$, then $P_{i, k-1}$ looses its connection with $\lambda_{k-1}$ and the pair $\left(u_{k-1}, v_{k-1}\right)$ can no longer be interpreted in terms of eigenvectors corresponding to $\lambda_{k-1}$. For small $\epsilon$ the sequence of estimates of the pseudospectral abscissa, generated by Algorithm 2, is always monotone close to the fixed-point, hence, iterations of Algorithm 3 reduce to iterations of Algorithm 2.

In what follows we illustrate two benefits of the adaptation. With Example 2 we illustrate how convergence to an attractor different from a fixed-point can be avoided. With Example 3 we show that the fixed point corresponding to the pseudospectral abscissa, may be unstable, but is still found using Algorithm 3.

EXAMPLE 2. We reconsider eigenvalue problem (4.12)-(4.14) where we take $\epsilon=5$ and $\left(w_{1}, w_{2}\right)=(1,+\infty)$. In Figure 5.1 we show iterations of Algorithm 2 (green curves) and Algorithm 3 (blue curves). While iterations of Algorithm 2 exhibit chaotic behavior, iterations of Algorithm 3 converge to the correct fixed point, characterized by $\alpha_{\epsilon}=1.2542565$. In Figure 5.2 the convergence of Algorithm 3 is visualized. Note that although $\epsilon$ is very large, the behavior of the error is similar to Example 1. 

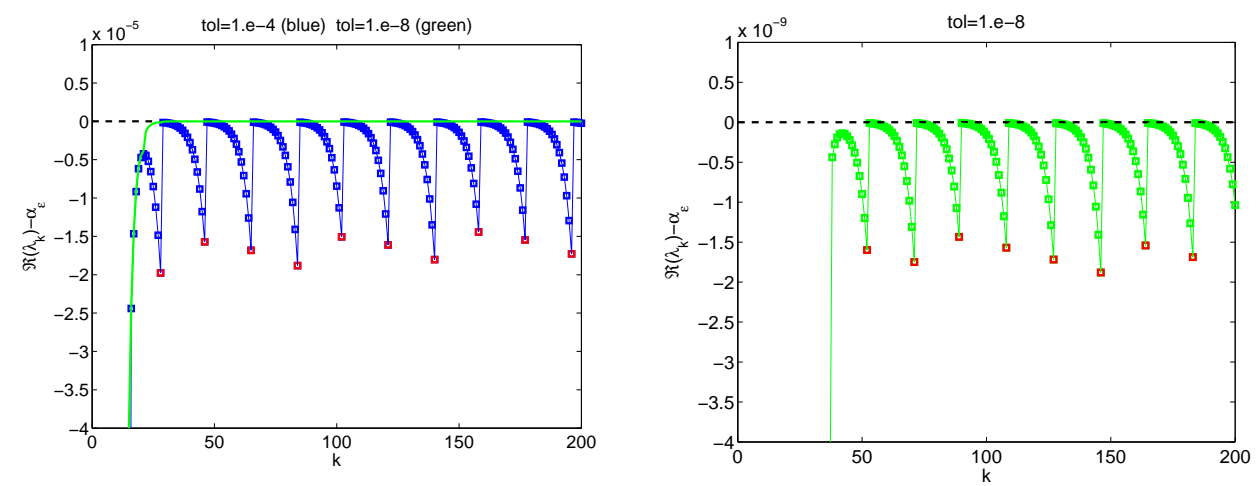

Fig. 5.3. The error, $\Re\left(\lambda_{k}\right)-\alpha_{\epsilon}$, as a function of $k$ for iterations of Algorithm 3 applied to Example 3. with tolerance tol $=10^{-4}$ (blue curve / squares) and tol $=10^{-8}$ (green curve / squares). Iterates for which (5.9) is satisfied are indicated with red squares. The right pane is a zoom of the left pane along the vertical axis. In order not to overload the figure, only iterations for tol $=10^{-8}$ are shown.

EXAMPle 3. We consider eigenvalue problem (4.12)-(4.14) with $\epsilon=4$ and, once again, $\left(w_{1}, w_{2}\right)=(1,+\infty)$. With the value tol $=10^{-8}$ in (5.9) Algorithm 3 seems to converge to a fixed point corresponding to the pseudospectral abscissa $\alpha_{\epsilon}=0.1885197$. However, repeating the experiment with larger values of the tolelance tol reveals that the fixed point is in fact locally unstable, yet Algorithm 3 converges to an attractor in the neighborhood of the fixed point, whose size can be controlled by the choice of the tolerance. Figure 5.3, which depicts the error $\Re\left(\lambda_{k}\right)-\alpha_{\epsilon}$ as a function of the iteration number, reveals the mechanism. As the fixed point is repulsive, iterations do not converge even when starting sufficiently close to it, which explains why the fixed point cannot be found by Algorithm 2. When using (the adapted) Algorithm 3 instead, the non converging phase stops on the moment when condition (5.9) is satisfied (indicated with the red boxes). At that point the mechanism to enforce an increase in the real part becomes active. As a consequence $\Re\left(\lambda_{k}\right)$ makes a jump, and the whole process repeats itself.

The above stabilization mechanism has a parallel with the notion of practical stabilization in the control literature (see, e.g. [19]): although the fixed point is not stabilized, the iteration can be forced to converge to an attractor contained in an arbitrarily small neighborhood of the fixed point.

REMARK 5.1. Recall that the basic Algorithm 1 is grounded in a mathematical characterization of rightmost points of a pseudospectrum contour, while Algorithm 2 and Algorithm 3 are essentially adaptations, to make an iteration well defined in all possible cases and to enforce global convergence to a fixed point, respectively. At this moment we have no theoretical proof that the sequence $\left\{\lambda_{k}\right\}_{k \geq 0}$ generated by Algorithm 3 converges to a rightmost point of the pseudospectrum in all possible cases (e.g., although we have never encountered the situation, it cannot a priori be excluded that Algorithm 3 converges to a fixed-point where (5.11) is not zero). However, it should be noted that the necessary conditions expressed by (3.10)-(3.14) can always be tested, while for problems to which level set algorithms apply, global optimality can be guaranteed by one iteration of such an algorithm.

5.2. Using different starting values. If there are several eigenvalues at the right part of the spectrum with real parts close to each other, it might happen that, 
due to a difference in the sensitivity, the rightmost point of the $\epsilon$-pseudospectrum is not related to a perturbation of the rightmost eigenvalue. This may cause convergence to a locally but not globally rightmost point. A way to avoid this consists of running Algorithm 3 several times, initialized with several eigenvalues with a large real part and/or a high sensitivity (instead of initialized with the rightmost eigenvalue only). The modification consists of an alternative choice of $\lambda_{1}$. As a measure of the local sensitivity of an eigenvalue $\lambda$, with corresponding eigenpair $(u, v)$, one can compute $\left(u^{*}\left(\sum_{i=1}^{m} A_{i} p_{i}^{\prime}(\lambda)\right) v\right)^{-1}$, which generalizes the notion of eigenvalue condition number.

Example 4. Consider system (4.12)-(4.14) with $\left(w_{1}, w_{1}\right)=(1, \infty)$ and $\epsilon=3.5$. The red lines in Figure 5.4 connect eigenvalues with points on the pseudospectrum contour, reached when initializing Algorithm 2 with the eigenvalue under consideration. It this case, the globally rightmost point of the pseudospectrum is not reached from the rightmost eigenvalue of the unperturbed system, but it is reached from another dominant eigenvalue.

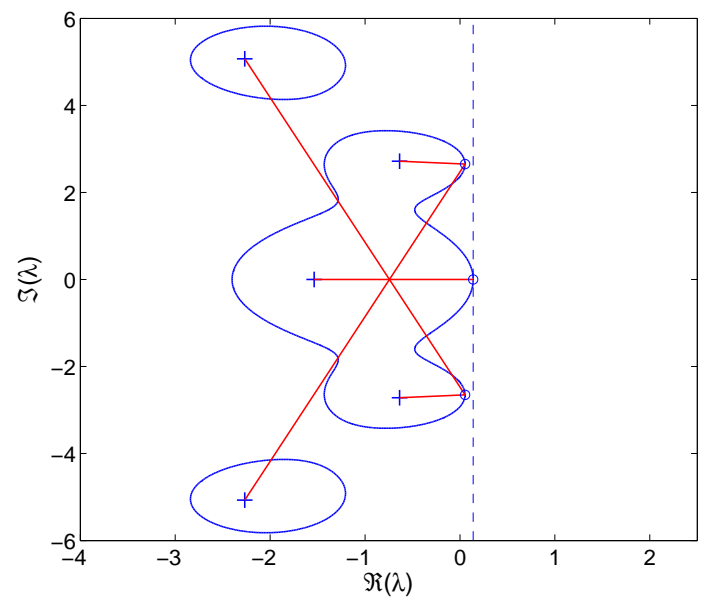

FIG. 5.4. Effect of an initialization with different eigenvalues.

6. Numerical experiments. We illustrate results of a MATLAB implementation of Algorithm 3 on polynomial and delay eigenvalue problems. For polynomial eigenvalue problems the eigenvalue computations are done using the function polyeig for small and medium-size problems and using eigs applied to a linearization in the other case. For delay eigenvalue problems the rightmost eigenvalues are computed using the algorithm described in [14], which can be interpreted as the Arnoldi method applied to an infinite-dimensional linear eigenvalue problem equivalent to the nonlinear delay eigenvalue problems. In all cases sparsity in the matrices can be exploited.

For both type of eigenvalue problems, the left eigenvectors are computed from the right eigenvectors of the dual system (obtained by replacing the system matrices by their complex conjugate transpose). In case of sparse matrices linear system solves are done using a sparse LU decomposition. In order to solve repeatedly a system of equations of the form

$$
\left(A+\epsilon \frac{p_{i}(\lambda)}{w_{i}\left|p_{i}(\lambda)\right|} u v^{*}\right) x=b_{i}
$$




\begin{tabular}{|l|l|r|r|}
\hline$\epsilon$ & $\alpha$ & $\alpha_{\epsilon}$ & \#it (10 digits) \\
\hline $10^{-3}$ & $9.472172578 \mathrm{e}-2$ & $9.565077802 \mathrm{e}-2$ & 3 \\
$10^{-2.8}$ & & $9.619554161 \mathrm{e}-2$ & 3 \\
$10^{-2.6}$ & & $9.706100877 \mathrm{e}-2$ & 3 \\
$10^{-2.4}$ & & $9.843791771 \mathrm{e}-2$ & 4 \\
$10^{-2.0}$ & & $1.041467015 \mathrm{e}-1$ & 4 \\
$10^{-1.8}$ & & $1.098009209 \mathrm{e}-1$ & 5 \\
$10^{-1.6}$ & & $1.189844647 \mathrm{e}-1$ & 5 \\
$10^{-1.4}$ & & $2.046203606 \mathrm{e}-1$ & 6 \\
$10^{-1.2}$ & & $9.017386843 \mathrm{e}-1$ & 7 \\
$10^{-1.0}$ & & $2.303505709+00$ & 8 \\
$10^{-0.8}$ & & $9.258176653+00$ & 10 \\
\hline \multicolumn{2}{|c}{ TABLE 6.1 } \\
\hline
\end{tabular}

Example 4.1 of [21]. The second column displays the spectral abscissa, the third one the pseudospectral abscissa and the last one the number of iterations needed for 10 digits accuracy.

\begin{tabular}{|l|l|r|r|r|}
\hline$\epsilon$ & $\alpha$ & $\alpha_{\epsilon}$ & \#it (10 digits) & \#it (3 digits) \\
\hline $10^{-4}$ & $2.116899507 \mathrm{e} 4$ & $2.117201634 \mathrm{e}+4$ & 2 & \\
\hline $10^{-3}$ & & $2.119925782 \mathrm{e}+4$ & 3 & 2 \\
\hline $10^{-2}$ & & $2.147691138 \mathrm{e}+4$ & 6 & 6 \\
\hline $10^{-1}$ & & $2.522301267 \mathrm{e}+4$ & 122 & \\
\hline \multicolumn{4}{|r|}{ TABLE 6.2}
\end{tabular}

Quadratic eigenvalue problem of size $n=3627$ from the NLEVP collection.

where only the rank-one term and the righthand side change, the Sherman-Morrison formula is used.

In accordance with the phenomenon described in 5.2 the algorithm is repeatedly run starting with three rightmost eigenvalues.

6.1. Polynomial eigenvalue problems. We consider Example 4.1 of [21], which is a quadratic eigenvalue problem of the form (2.1) where $m=3$,

$A_{1}=\left[\begin{array}{ccc}121 & 18.9 & 15.9 \\ 0 & 2.7 & 0.145 \\ 11.9 & 3.64 & 15.5\end{array}\right], A_{2}=\left[\begin{array}{ccc}7.66 & 2.45 & 2.1 \\ 0.23 & 1.04 & 0.223 \\ 0.6 & 0.756 & 0.658\end{array}\right], A_{3}=\left[\begin{array}{ccc}17.6 & 1.28 & 2.89 \\ 1.28 & 0.824 & 0.412 \\ 2.89 & 0.413 & 0.725\end{array}\right]$.

When applying perturbations to all system matrices and taking unity weights, i.e.

$$
\left(w_{1}, w_{2}, w_{2}\right)=(1,1,1)
$$

the results of the computations are displayed in Table 6.1. The results are in accordance with the pseudospectra contours visualized in [21].

A second example has been taken from the NLEVP collection [1]. It corresponds to the problem 'foundation', which is also a quadratic eigenvalue problem. The system matrices are sparse with dimension $n=3627$. With weights chosen as $\left(w_{1}, w_{2}, w_{3}\right)=$ $\left(1 /\left\|A_{1}\right\|_{2}, 1 /\left\|A_{2}\right\|_{2}, \infty\right)$, results of the pseudospectral abscissa computation are shown in Table 6.2.

6.2. Delay eigenvalue problem. First, we have applied Algorithm 3 to the benchmark collection ${ }^{3}$ used in [12] These consists of problems of the form (2.2). The

\footnotetext{
${ }^{3}$ the collection is available at the webpage http://twr.cs.kuleuven.be/research/software/delaycontrol/benchmarks/
} 


\begin{tabular}{|c|c|c|c|c|c|c|}
\hline Problem & $(n, \#$ delays $)$ & $\alpha$ & $\epsilon$ & $\alpha_{\epsilon}$ & $\begin{array}{r}\text { \#it } \\
\text { (10 digits) }\end{array}$ & $\begin{array}{r}\text { \#it } \\
(3 \text { digits })\end{array}$ \\
\hline \multirow[t]{3}{*}{1} & \multirow{3}{*}{$(3,1)$} & \multirow{3}{*}{$-2.866038425 \mathrm{e}-02$} & 1.e-4 & $-2.853917009 \mathrm{e}-2$ & 3 & 2 \\
\hline & & & 1.e-2 & $-1.716339042 \mathrm{e}-2$ & 4 & 2 \\
\hline & & & 1.e-1 & $5.607794107 \mathrm{e}-2$ & 8 & 4 \\
\hline \multirow[t]{3}{*}{2} & \multirow[t]{3}{*}{$(1,1)$} & \multirow[t]{3}{*}{$-1.102659477+00$} & 1.e-4 & $-1.102516288+00$ & 3 & 1 \\
\hline & & & 1.e-2 & $-1.088390378+00$ & 3 & 2 \\
\hline & & & 1.e-1 & $-9.641158746 \mathrm{e}-1$ & 5 & 2 \\
\hline \multirow[t]{3}{*}{3} & \multirow[t]{3}{*}{$(3,3)$} & \multirow[t]{3}{*}{$-2.944578327 \mathrm{e}-01$} & 1.e-4 & $-2.874841199 \mathrm{e}-1$ & 3 & 2 \\
\hline & & & 1.e-2 & $3.591092039 \mathrm{e}-1$ & 7 & 3 \\
\hline & & & 1.e-1 & $4.275010482+00$ & 29 & 11 \\
\hline \multirow[t]{3}{*}{4} & \multirow[t]{3}{*}{$(4,9)$} & \multirow[t]{3}{*}{$1.169539686+00$} & 1.e-4 & $1.174506091+00$ & 3 & 1 \\
\hline & & & 1.e-2 & $1.674277721+00$ & 115 & 1 \\
\hline & & & 1.e-1 & $3.912462373+00$ & 35 & 11 \\
\hline \multirow[t]{3}{*}{5} & \multirow[t]{3}{*}{$(8,20)$} & \multirow[t]{3}{*}{$2.373606203+00$} & $1 . \mathrm{e}-4$ & $2.374682455+00$ & 2 & 1 \\
\hline & & & 1.e-2 & $2.480435391+00$ & 5 & 2 \\
\hline & & & 1.e-1 & $3.406557005+00$ & 170 & 23 \\
\hline \multirow[t]{3}{*}{6} & \multirow[t]{3}{*}{$(10,7)$} & \multirow[t]{3}{*}{$-3.775473572 \mathrm{e}-01$} & $1 . \mathrm{e}-4$ & $-3.759673125 \mathrm{e}-1$ & 3 & 2 \\
\hline & & & 1.e-2 & $-2.301342421 \mathrm{e}-1$ & 7 & 3 \\
\hline & & & 1.e-1 & $1.046143337+00$ & 123 & 40 \\
\hline \multirow[t]{3}{*}{7} & \multirow[t]{3}{*}{$(20,9)$} & \multirow[t]{3}{*}{$-3.446892131 \mathrm{e}-02$} & 1.e-4 & $-3.290058652 \mathrm{e}-2$ & 4 & 2 \\
\hline & & & 1.e-2 & $8.291146618 \mathrm{e}-2$ & 18 & 6 \\
\hline & & & 1.e-1 & $1.182937400+00$ & 60 & 9 \\
\hline \multirow[t]{3}{*}{8} & \multirow[t]{3}{*}{$(40,3)$} & \multirow[t]{3}{*}{$-1.044598769 \mathrm{e}-01$} & 1.e-4 & $-1.027893926 \mathrm{e}-1$ & 3 & 2 \\
\hline & & & 1.e-2 & $3.015727003 \mathrm{e}-2$ & 13 & 5 \\
\hline & & & 1.e-1 & $8.398294386 \mathrm{E}-1$ & 265 & 20 \\
\hline \multirow[t]{3}{*}{9} & \multirow[t]{3}{*}{$(5,1)$} & \multirow[t]{3}{*}{$-5.026086111 \mathrm{e}-01$} & 1.e-4 & $-5.009786768 \mathrm{e}-1$ & 3 & 2 \\
\hline & & & 1.e-2 & $-3.474748581 \mathrm{e}-1$ & 7 & 4 \\
\hline & & & 1.e-1 & $7.743617965 \mathrm{e}-1$ & 50 & 11 \\
\hline \multirow[t]{3}{*}{10} & \multirow[t]{3}{*}{$(4,3)$} & \multirow[t]{3}{*}{$-9.85848814 \mathrm{e}-02$} & 1.e-4 & $-9.853953913 \mathrm{e}-2$ & 2 & 2 \\
\hline & & & 1.e-2 & $-9.402496447 \mathrm{e}-2$ & 7 & 2 \\
\hline & & & 1.e-1 & $-2.026579099 \mathrm{e}-2$ & 26 & 10 \\
\hline
\end{tabular}

TABLE 6.3

Pseudospectral abscissa computation for delay eigenvalue problems from the same benchmark set used in [12].

weights are chosen as

$$
w_{i}=\left\|B_{i}\right\|_{2}^{-1}, 1 \leq i \leq m
$$

i.e. the value of $\epsilon$ refers to the maximal relative size of the perturbations. The results are displayed in Table 6.3 and they have been validated with the level method described in [12].

To demonstrate the applicability to a large-scale problem we consider the PDE with delay from [14],

$$
\frac{\partial v(x, t)}{\partial t}=\frac{\partial^{2} v(x, t)}{\partial x^{2}}+a_{0}(x) v(x, t)+a_{1}(x) v(\pi-x, t-1) .
$$

where $a_{0}(x)=-2 \sin (x), a_{1}(x)=2 \sin (x)$ and $v_{x}(0, t)=v_{x}(\pi, t)=0$. The second derivatives in space in are approximated with central differences. This gives rise to a standard delay eigenvalue problem of the form (2.2), with one delay and sparse 


\begin{tabular}{|r|r|r|r|r|r|}
\hline$(n$, \#delays $)$ & $\alpha$ & $\left(w_{1}, w_{2}\right)$ & $\epsilon$ & $\alpha_{\epsilon}$ & $\#$ it \\
\hline$(5000,1)$ & $-3.312133337 \mathrm{e}-1$ & $(1 / 2,1 / 2)$ & $1 . \mathrm{e}-3$ & $-3.297978515 \mathrm{e}-1$ & 2 \\
& & & $1 . \mathrm{e}-2$ & $-3.170982221 \mathrm{e}-1$ & 3 \\
& & & $1 . \mathrm{e}-1$ & $-1.937166436 \mathrm{e}-1$ & 4 \\
& & & $1+00$ & $8.647127140 \mathrm{e}-1$ & 8 \\
\hline & & $(1 / 4, \infty)$ & $1 . \mathrm{e}-3$ & $-3.300292687 \mathrm{e}-1$ & 2 \\
& & $1 . \mathrm{e}-2$ & $-3.193270916 \mathrm{e}-1$ & 3 \\
& & $1 . \mathrm{e}-1$ & $-2.075848221 \mathrm{e}-1$ & 4 \\
& & & $1+00$ & $+1.641134830 \mathrm{e} 00$ & 10 \\
\hline & & $(\infty, 1 / 4)$ & $1 . \mathrm{e}-3$ & $-3.295667765 \mathrm{e}-1$ & 2 \\
& & & $1 . \mathrm{e}-2$ & $-3.149018637 \mathrm{e}-1$ & 3 \\
& & & $1 . \mathrm{e}-1$ & $-1.816328080 \mathrm{e}-1$ & 4 \\
& & & $1+00$ & $+5.599912563 \mathrm{e}-1$ & 7 \\
\hline
\end{tabular}

Pseudospectral abscissa for the delayed PDE problem described in [14].

matrices $B_{0}$ and $B_{1}$. The number of spatial discretization points is taken such that $n=5000$. The results are displayed in Table 6.4.

7. Concluding remarks. The presented Algorithms 2-3 for the pseudospectral abscissa computationcan be applied to the broad class of nonlinear eigenvalue problems (1.1). The algorithms only require a procedure to compute selected (rightmost) eigenvalues and corresponding eigenvectors. This makes them particularly well suited to large-scale sparse problems whenever fast iterative eigensolvers are available. The effectiveness of the proposed approach is further illustrated by the fact that for most nonlinear eigenvalue problems standard level set methods like [6] are not applicable.

In many algorithms for solving nonlinear eigenvalue problems, the connection with a linearization of the eigenvalue problem plays an important role. In this sense the adopted approach for the pseudospectral abscissa computation fully exploits the dual representation of the eigenvalue problem: on the one hand pseudospectra are defined at the level of the nonlinear problem in such a way that the structure of the problem and the perturbations are respected; on the other hand the corresponding algorithm relies on the successive computation of selected eigenvalues, for which the connection with the linearization may be very beneficial.

For small values of $\epsilon$, the convergence of Algorithm 2 has been rigorously proved and the convergence properties have been analyzed. For the special case of a linear eigenvalue problem the same conclusions are reached as in [11], but using different arguments. Algorithm 3 also performs very well for large values of $\epsilon$. It includes several modifications to handle situations where Algorithm 2 does not converge. It should be noted, however, that such situations are rarely observed in experiments. For the illustrative examples in Section 5 the parameters of the examples were carefully chosen to "trigger" the special situations, and the perturbations had norm at least $50 \%$ of the norm of the matrices.

Similarly to the case of the standard eigenvalue problem the algorithm can be adapted to solve related problems such as the computation of the pseudospectral radius, $\mathcal{H}_{\infty}$ norms and complex stability radii. Future work also includes handling (additional) structure on the perturbations of the individual coefficient matrices $A_{i}$ and using the algorithm in the context of stability optimization, with applications in robust control. 
Appendix A. Other perturbation measure. In [17] also other measures of the perturbation than (1.3) are considered. These include

$$
\|\Delta\|_{\text {glob }}:=\left\|\left[\begin{array}{c}
w_{1} \delta A_{1} \\
\vdots \\
w_{m} \delta A_{m}
\end{array}\right]\right\|_{2}, \quad\|\Delta\|_{\text {glob }}:=\left\|\left[\begin{array}{lll}
w_{0} \delta A_{0} & \cdots & w_{m} \delta A_{m}
\end{array}\right]\right\|_{2} .
$$

For both measures (A.1) it was shown that the corresponding pseudospectra satisfy

$$
\Lambda_{\epsilon}=\left\{\lambda \in \mathbb{C}: \sigma_{n}\left(\sum_{i=1}^{m} A_{i} p_{i}(\lambda)\right) \leq \epsilon\|w(\lambda)\|_{2}\right\} .
$$

The results in the previous sections and the algorithms can be adapted accordingly. In particular, the "critical perturbations" (3.1) become

$$
\delta A_{i}=-\frac{\overline{p_{i}\left(\lambda_{\epsilon}\right)}}{w_{i}^{2} \sqrt{\sum_{k=1}^{m} \frac{\left|p_{k}\left(\lambda_{\epsilon}\right)\right|^{2}}{w_{k}^{2}}}} u v^{*} \epsilon, \quad 1 \leq i \leq m,
$$

and the optimality conditions (3.5)-(3.6) are changed to

$$
\begin{aligned}
& \Im\left(u^{*}\left(\sum_{i=1}^{m} A_{i} p_{i}^{\prime}\left(\lambda_{\epsilon}\right)\right) v\right)-\frac{\epsilon}{\sqrt{\sum_{i=1}^{m} \frac{\left|p_{i}\left(\lambda_{\epsilon}\right)\right|^{2}}{w_{i}^{2}}}} \sum_{k=1}^{m} \frac{\Im\left(\overline{p_{k}\left(\lambda_{\epsilon}\right)} p_{k}^{\prime}\left(\lambda_{\epsilon}\right)\right)}{w_{k}^{2}}=0, \\
& \Re\left(u^{*}\left(\sum_{i=1}^{m} A_{i} p_{i}^{\prime}\left(\lambda_{\epsilon}\right)\right) v\right)-\frac{\epsilon}{\sqrt{\sum_{i=1}^{m} \frac{\left|p_{i}\left(\lambda_{\epsilon}\right)\right|^{2}}{w_{i}^{2}}}} \sum_{k=1}^{m} \frac{\Re\left(\overline{p_{k}\left(\lambda_{\epsilon}\right)} p_{k}^{\prime}\left(\lambda_{\epsilon}\right)\right)}{w_{k}^{2}}>0 .
\end{aligned}
$$

Acknowledgments. N.G. wishes to thank the Department of Computer Science of the University of Leuven for the kind hospitality. This work has been supported by the Programme of Interuniversity Attraction Poles of the Belgian Federal Science Policy Oce (IAP P6- DYSCO), by OPTEC, the Optimization in Engineering Center of the K.U.Leuven, by the project STRT1- 09/33 of the K.U.Leuven Research Council and the project G.0712.11N of the Research Foudation - Flanders (FWO) and by the Italian Ministry of Education, Universities and Research (M.I.U.R.) and Gruppo Nazionale di Calcolo Scientifico (G.N.C.S.).

\section{REFERENCES}

[1] T. Betcke, N. J. Higham, V. Mehrmann, C. Schrder, and F. Tisseur. NLEVP: A collection of nonlinear eigenvalue problems. Technical Report EPrint 2010.98, Manchester Institute for Mathematical Sciences, 2010.

[2] S. Boyd and V. Balakrishnan. A regularity result for the singular values of a transfer matrix and a quadratically convergent algorithm for computing its $\mathcal{L}_{\infty}$-norm. Systems $\&$ Control Letters, 15:1-7, 1990 .

[3] D. Breda, S. Maset, and R. Vermiglio. Pseudospectral differencing methods for characteristic roots of delay differential equations. SIAM Journal on Scientific Computing, 27(2):482495, 2005.

[4] N.A. Bruinsma and M. Steinbuch. A fast algorithm to compute the $\mathcal{H}_{\infty}$-norm of a transfer function matrix. Systems and Control Letters, 14:287-293, 1990.

[5] J.V. Burke, A.S. Lewis, and M.L. Overton. Optimization and pseudospectra, with applications to robust stability. SIAM Journal on Matrix Analysis and Applications, 25(1):80-104, 2003. 
[6] J.V. Burke, A.S. Lewis, and M.L. Overton. Robust stability and a criss-cross algorithm for pseudospectra. IMA Journal of Numerical Analysis, 23:359-375, 2003.

[7] R. Byers. A bisection method for measuring the distance of a stable matrix to the unstable matrices. SIAM Journal on Scientific and Statistical Computing, 9(9):875-881, 1988.

[8] K. Engelborghs, T. Luzyanina, and G. Samaey. DDE-BIFTOOL v. 2.00: a Matlab package for bifurcation analysis of delay differential equations. TW Report 330, Department of Computer Science, Katholieke Universiteit Leuven, Belgium, October 2001.

[9] K. Gu, V.L. Kharitonov, and J. Chen. Stability of time-delay systems. Birkhauser, 2003.

[10] N. Guglielmi and C. Lubich. Differential equations for roaming pseudospectra: paths to extremal points and boundary tracking. SIAM Journal on Numerical Analysis, 49:1194-1209, 2011.

[11] N. Guglielmi and M.L. Overton. Fast algorithms for the approximation of the pseudospectral abscissa and pseudospectral radius of a matrix. SIAM Journal on Matrix Analysis and Applications, 32:1166-1192. In Press.

[12] S. Gumussoy and W. Michiels. A predictor-corrector type algorithm for the pseudospectral abscissa computation of time-delay systems. Automatica, 46(4):657-664, 2010.

[13] R. Hryniv and P. Lancaster. On the perturbation of analytic matrix functions. Integral Equations and Operator Theory, 34:325-338, 1999.

[14] E. Jarlebring, K. Meerbergen, and W. Michiels. A krylov method for the delay eigenvalue problem. SIAM Journal on Scientific Computing, 32(6):3278-3300, 2010.

[15] E. Jarlebring, W. Michiels, and K. Meerbergen. A linear eigenvalue algorithm for the nonlinear eigenvalue problem. TW Report 580, Department of Computer Science, Katholieke Universiteit Leuven, Belgium, October 2010.

[16] V. Mehrmann and H. Voss. Nonlinear eigenvalue problems: A challenge for modern eigenvalue methods. GAMM Mitteilungen, 27:121-152, 2004.

[17] W. Michiels, K. Green, T. Wagenknecht, and S.-I. Niculescu. Pseudospectra and stability radii for analytic matrix functions with applications to time-delay systems. Linear Algebra and its Applications, 418(1):315-335, 2006.

[18] W. Michiels and S.-I. Niculescu. Stability and stabilization of time-delay systems. An eigenvalue based approach. SIAM, 2007.

[19] L. Moreau and D. Aeyels. Practical stability and stabilization. IEEE Transactions on Automatic Control, 45(8):1554-1558, 2000.

[20] J. Nocedal and S.J. Wright. Numerical Optimization. Springer Series in Operations Research. Springer-Verlag New York, 1999.

[21] F. Tisseur and N. J. Higham. Structured pseudospectra for polynomial eigenvalue problems, with applications. SIAM J. Matrix Anal. Appl., 23(1):187-208, 2001.

[22] F. Tisseur and K. Meerbergen. The quadratic eigenvalue problem. SIAM Rev., 43(2):235-286, 2001.

[23] L.N. Trefethen and M. Embree. Spectra and pseudospectra: the behavior of nonnormal matrices and operators. Princeton University Press, 2005.

[24] H. Voss. An Arnoldi method for nonlinear eigenvalue problems. BIT, 44:387 - 401, 2004.

[25] H. Voss. A new justification of the jacobi-davidson method for large eigenproblems. Linear Algebra Appl., 424:448-455, 2007.

[26] K. Zhou, J.C. Doyle, and K. Glover. Robust and optimal control. Prentice Hall, 1995. 